\title{
Wave propagation through Cantor-set media: Chaos, scaling, and fractal structures
}

\author{
Kenta Esaki, Masatoshi Sato, and Mahito Kohmoto \\ Institute for Solid State Physics, Kashiwanoha 5-1-5, Kashiwa, Chiba, 277-8581, Japan
}

(Dated: September 29, 2018)

\begin{abstract}
Propagation of waves through Cantor-set media is investigated by renormalization-group analysis. For specific values of wave numbers, transmission coefficients are shown to be governed by the logistic map, and in the chaotic region, they show sensitive dependence on small changes of parameters of the system such as the index of refraction. For other values of wave numbers, our numerical results suggest that light transmits completely or reflects completely by the Cantor-set media $\mathrm{C}_{\infty}$. It is also shown that transmission coefficients exhibit a local scaling behavior near complete transmission if the complete transmission is achieved at a wave number $\kappa=\kappa^{*}$ with a rational $\kappa^{*} / \pi$. The scaling function is obtained analytically by using the Euler's totient function, and the local scaling behavior is confirmed numerically.
\end{abstract}

PACS numbers: 05.45.-a, 42.25.-p, 61.44.-n 


\section{INTRODUCTION}

Wave properties in fractal [1, 2, 3, 4, 5, 6, 7, 8, 9, 10, 11] and quasi-periodic [12, 13, 14, 15, 16, 17] structures in one dimension have been of theoretical and practical interest over the past two decades. They are typical examples of self-similar structures, and physical properties peculiar to them have been explored. In order to observe effects of quasiperiodicity, optical experiments using dielectric multilayers of $\mathrm{SiO}_{2}$ and $\mathrm{TiO}_{2}$ films were performed for the Fibonacci multilayer [16, 17]. In these experiments, scaling behaviors of the transmission coefficients were observed, which had been predicted by the renormalization-group theory [15]. For fractal structures, optical wave propagation on Cantor multilayers has been studied by several authors [2, 3, 4, 5, 6, 7, 8, 9]. Self-similar structures of the transmission (or reflection) coefficients were obtained numerically [2, 3, 4, 4, 5, 6, 7]. Moreover, resonant states of light were studied [8, 9], which was motivated by an experiment using a three-dimensional fractal cavity called the Menger sponge [18].

In this paper, we reexamine the optical wave propagation through Cantor sequences on the basis of the renormalization-group theory. In addition to the self-similar structures mentioned above, we find interesting chaotic behaviors of the transmission coefficients analytically. It is well known that non-linear dynamical systems showing chaotic behaviors often have strange attractors with fractal structures[19], but we show that the reverse is also possible. Namely, chaotic dynamics is obtained from a fractal structure. In the following, we find that, for specific values of wave numbers, the transmission coefficients show chaotic behaviors governed by the logistic map. For these wave numbers, the transmission coefficients are very sensitive to small changes of parameters of the system such as the index of refraction. This exotic behavior leads to rapid oscillations of the transmission coefficients as functions of the index of refraction, which could be observed in an optical experiment. For other wave numbers, our numerical study suggests that light eventually transmits completely or reflects completely for the infinite generation of the Cantor sequences $\mathrm{C}_{\infty}$.

We will also find intriguing local scaling behaviors of the transmission coefficients near complete transmission, which are distinct from the self-similar structures found in Refs. [2, 3, 4]. The complete transmission can be regarded as a fixed point of the renormalization-group equation, and the local scaling behaviors are obtained on the basis of the renormalizationgroup theory. It will be shown that if complete transmission is achieved at a wave number 


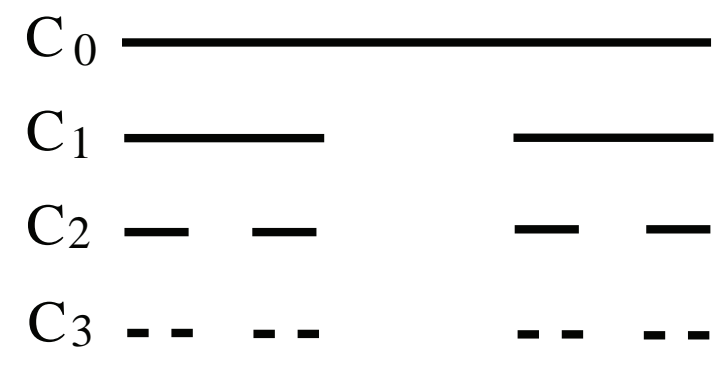

FIG. 1: The first four generations of the Cantor sequences $\mathrm{C}_{j}$. The Cantor-set media $\mathrm{C}_{\infty}$ are obtained in the $j \rightarrow \infty$ limit.

$\kappa=\kappa^{*}$ with a rational $\kappa^{*} / \pi$, the transmission coefficient around the wave number exhibits a local scaling behavior. The scaling relation is determined by the rational number $\kappa^{*} / \pi$ through the Euler's totient function, and an analytic expression of the scaling function will be presented. We will also compare the analytical results with numerical data.

This paper is organized as follows. In Sec,II, we briefly explain the Cantor sequences and the Cantor-set media. We then formulate our problem in terms of renormalizationgroup transformation. In Sec III, we classify wave propagation and find chaotic behaviors of transmission coefficients governed by the logistic map for specific wave numbers. Optical experiments to observe the chaotic behaviors are also proposed. For other wave numbers, complete transmission and complete reflection of light in the Cantor-set media $\mathrm{C}_{\infty}$ are numerically suggested. In Sec IV, scaling behaviors of the transmission coefficients near complete transmission are analyzed on the basis of the renormalization-group equation. Finally, in Sec $\mathrm{V}$, we summarize our results and discuss a generalization of our analysis for generalized Cantor-set media.

\section{TRANSFER-MATRIX METHOD AND RENORMALIZATION-GROUP TRANSFORMATION}

In this section, we make a formulation of our problem. The Cantor sequences and the Cantor-set media are constructed, and the transfer matrix method is introduced to study wave propagation through them. Then the renormalization-group equation is defined in terms of the transfer matrix method.

Let us construct the Cantor-set media first. See Fig.1. The procedure of constructing 
the Cantor set begins with a line segment with unit length $\left(\mathrm{C}_{0}\right.$ in Fig 1$)$. We regard this as substrate $\mathrm{A}$. To obtain the first generation $\mathrm{C}_{1}$, the line segment is divided into three parts. The left and the right segments are substrate A, each of which has length $1 / 3$, and the middle part, which has length $1 / 3$, is removed. We regard the removed part as substrate B. Then the procedure is repeated for each of remaining line segments A to obtain new generations. We call the $j$ th generation of the Cantor sequence as $\mathrm{C}_{j}$. For $\mathrm{C}_{j}$ we have a set of $2^{j}$ line segments of substrate $\mathrm{A}$, each of which has length $1 / 3^{j}$. By repeating this procedure infinite times, we finally obtain the Cantor-set media, $\mathrm{C}_{\infty}$, which are self-similar and have fractal dimension $\log 2 / \log 3$. In the following, we denote the indices of refraction of $\mathrm{A}$ and $\mathrm{B}$ as $n_{\mathrm{A}}$ and $n_{\mathrm{B}}$, respectively, and take $n_{\mathrm{B}}=1$ without loss of generality.

Now consider wave propagation through the Cantor sequence $\mathrm{C}_{j}$ illustrated in Fig,2, For simplicity, we suppose that the incident light is linearly polarized. Here $E_{\mathrm{L}}^{(1)}, E_{\mathrm{L}}^{(2)}$ and $E_{\mathrm{R}}^{(1)}$ denote the incident light, the reflected light and the transmitted light, respectively. No incoming wave from the right $E_{\mathrm{R}}^{(2)}$ exists, $E_{\mathrm{R}}^{(2)}=0$. In order to understand light propagation through $\mathrm{C}_{j}$, let us first consider interfaces of two layers in Fig. 3 . The electric field for light in layer $\mathrm{A}$ is given by

$$
E=E_{\mathrm{A}}^{(1)} \exp \left[i k_{\mathrm{A}} x-i \omega t\right]+E_{\mathrm{A}}^{(2)} \exp \left[-i k_{\mathrm{A}} x-i \omega t\right]
$$

where $k_{\mathrm{A}}=n_{\mathrm{A}} k$ is the wave number of light in substrate $\mathrm{A}$ and $\omega$ is frequency of light. ( $k$ is a wave number of light in the vacuum.) The electric field in a layer B is given by the same expression with the subscript A replaced by B. The boundary condition on the interface at the position $x=l$ is given by

$$
\begin{aligned}
& E_{\mathrm{A}}^{(1)} e^{i k_{\mathrm{A}} l}+E_{\mathrm{A}}^{(2)} e^{-i k_{\mathrm{A}} l}=E_{\mathrm{B}}^{(1)} e^{i k_{\mathrm{B}} l}+E_{\mathrm{B}}^{(2)} e^{-i k_{\mathrm{B}} l} \\
& n_{\mathrm{A}}\left(E_{\mathrm{A}}^{(1)} e^{i k_{\mathrm{A}} l}-E_{\mathrm{A}}^{(2)} e^{-i k_{\mathrm{A}} l}\right)=E_{\mathrm{B}}^{(1)} e^{i k_{\mathrm{B}} l}-E_{\mathrm{B}}^{(2)} e^{-i k_{\mathrm{B}} l} .
\end{aligned}
$$

By introducing the following variables

$$
E_{+}=E^{(1)}+E^{(2)}, \quad E_{-}=\left(E^{(1)}-E^{(2)}\right) / i,
$$

(2) is recast into

$$
\left(\begin{array}{c}
E_{+} \\
E_{-}
\end{array}\right)_{\mathrm{A}}=\mathcal{T}^{-1}\left(n_{\mathrm{A}} k l\right) \mathcal{T}_{\mathrm{AB}} \mathcal{T}(k l)\left(\begin{array}{c}
E_{+} \\
E_{-}
\end{array}\right)_{\mathrm{B}} .
$$




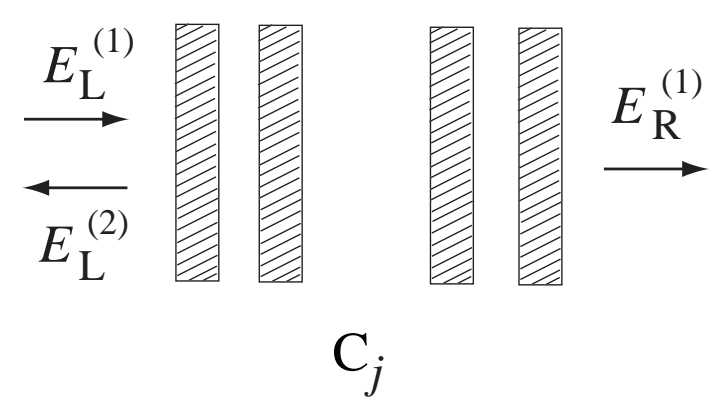

FIG. 2: Electromagnetic wave propagation through the Cantor sequence $\mathrm{C}_{j}(j=2)$.

Here $\mathcal{T}(\delta)$ and $\mathcal{T}_{\mathrm{AB}}$ are transfer matrices given by

$$
\mathcal{T}(\delta)=\left(\begin{array}{cc}
\cos \delta & -\sin \delta \\
\sin \delta & \cos \delta
\end{array}\right), \quad \mathcal{T}_{\mathrm{AB}}=\left(\begin{array}{cc}
1 & 0 \\
0 & 1 / n_{\mathrm{A}}
\end{array}\right),
$$

which represent light propagation within a layer and across an interface $A \leftarrow B$, respectively. In a similar manner, from the boundary condition on the interface at $x=l+d$, we have

$$
\left(\begin{array}{l}
E_{+} \\
E_{-}
\end{array}\right)_{\mathrm{B}^{\prime}}=\mathcal{T}^{-1}(k(l+d)) \mathcal{T}_{\mathrm{BA}} \mathcal{T}\left(n_{\mathrm{A}} k(l+d)\right)\left(\begin{array}{c}
E_{+} \\
E_{-}
\end{array}\right)_{\mathrm{A}},
$$

where $\mathcal{T}_{\mathrm{BA}}=\mathcal{T}_{\mathrm{AB}}^{-1}$ is the transfer matrix representing light propagation across an interface $B \leftarrow A$. Combining (4) with (6) , we obtain

$$
\left(\begin{array}{c}
E_{+} \\
E_{-}
\end{array}\right)_{\mathrm{B}^{\prime}}=\mathcal{T}^{-1}(k(l+d)) \mathcal{T}_{\mathrm{BA}} \mathcal{T}\left(n_{\mathrm{A}} k d\right) \mathcal{T}_{\mathrm{AB}} \mathcal{T}(k l)\left(\begin{array}{c}
E_{+} \\
E_{-}
\end{array}\right)_{\mathrm{B}} .
$$

Here note that, for a layer A with thickness $d$, the phase $\delta$ is given by $\delta=n_{\mathrm{A}} k d$, and for a layer $\mathrm{B}$ with thickness $d$, the phase $\delta$ is given by $\delta=k d$.

We are now ready to consider light propagation through $\mathrm{C}_{j}$ in Fig,2, Repeating the similar procedure above, we obtain the following formula for light propagation through $\mathrm{C}_{j}$.

$$
\left(\begin{array}{l}
E_{+} \\
E_{-}
\end{array}\right)_{\mathrm{R}}=e^{-i k} \mathcal{M}_{j}(k)\left(\begin{array}{l}
E_{+} \\
E_{-}
\end{array}\right)_{\mathrm{L}},
$$

where $E_{ \pm}$is defined by (3) for $E_{\mathrm{L}}^{(1)}, E_{\mathrm{L}}^{(2)}$ and $E_{\mathrm{R}}^{(1)}$. The real matrix $\mathcal{M}_{j}(k)$ is obtained recursively,

$$
\mathcal{M}_{j+1}(k)=\mathcal{M}_{j}(k / 3) \mathcal{T}(k / 3) \mathcal{M}_{j}(k / 3),
$$




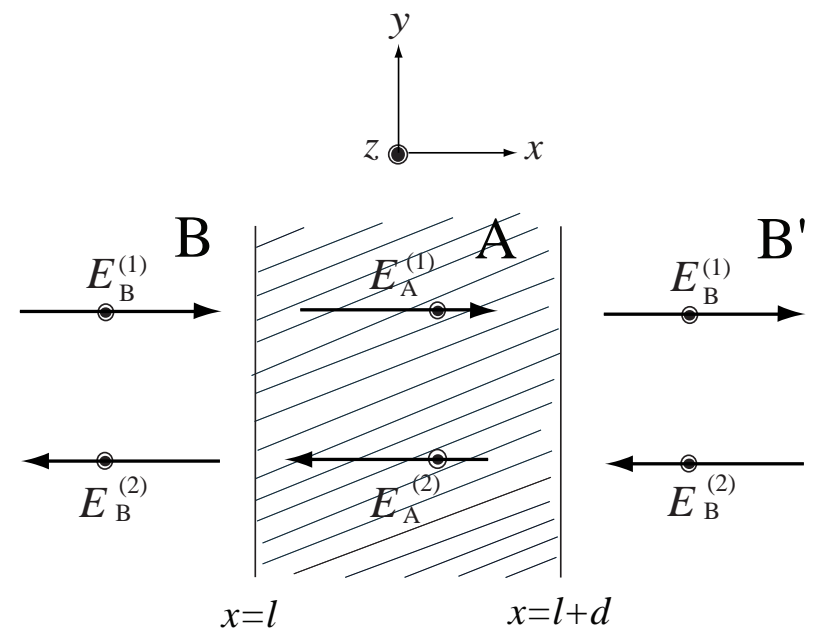

FIG. 3: Electromagnetic wave propagation across interfaces between two layers A and B.

with the initial condition $\mathcal{M}_{0}(k)=\mathcal{T}_{\mathrm{BA}} \mathcal{T}_{\mathrm{A}}\left(n_{\mathrm{A}} k\right) \mathcal{T}_{\mathrm{AB}}$. Using the initial condition and the recursive relation, we can show

$$
\operatorname{det} \mathcal{M}_{j}(k)=1, \quad\left(\mathcal{M}_{j}(k)\right)_{11}=\left(\mathcal{M}_{j}(k)\right)_{22}
$$

By eliminating $E_{L}^{(2)}$ from (일 , the ratio of the amplitude of the transmitted light $E_{R}^{(1)}$ to that of the incident light $E_{L}^{(1)}$ for $\mathrm{C}_{j}$ is obtained as

$$
\frac{E_{R}^{(1)}}{E_{L}^{(1)}}=\frac{2 e^{-i k}}{2 a_{j}+i\left(b_{j}-c_{j}\right)}
$$

where $a_{j}(k), b_{j}(k)$ and $c_{j}(k)$ are components of $\mathcal{M}_{j}(k)$

$$
\mathcal{M}_{j}(k)=\left(\begin{array}{cc}
a_{j}(k) & b_{j}(k) \\
c_{j}(k) & a_{j}(k)
\end{array}\right), \quad a_{j}(k)^{2}-b_{j}(k) c_{j}(k)=1 .
$$

Therefore the transmission coefficient $T_{j} \equiv\left|E_{\mathrm{R}}^{(1)}\right|^{2} /\left|E_{\mathrm{L}}^{(1)}\right|^{2}$ of $\mathrm{C}_{j}$ is given by

$$
T_{j}(k)=\frac{4}{\left|\mathcal{M}_{j}(k)\right|^{2}+2},
$$

where $\left|\mathcal{M}_{j}(k)\right|^{2} \equiv 2 a_{j}(k)^{2}+b_{j}(k)^{2}+c_{j}(k)^{2}$.

In Fig 4 we illustrate a typical example of transmission coefficients as functions of the wave number for $\mathrm{C}_{2}, \mathrm{C}_{3}$, and $\mathrm{C}_{4}$. As is shown clearly, we have a scaling behavior of the transmission coefficients: If we multiply the wave number by three as one generation increases, we have a similar structure in the transmission coefficients. To describe the scaling 
behavior properly, we introduce the rescaled wave number $k_{j}=3^{j} \kappa$ for $\mathrm{C}_{j}$, then from (9), we have

$$
\mathcal{M}_{j+1}\left(k_{j+1}\right)=\mathcal{M}_{j}\left(k_{j}\right) \mathcal{T}\left(3^{j} \kappa\right) \mathcal{M}_{j}\left(k_{j}\right), \quad \operatorname{det} \mathcal{M}_{j}\left(k_{j}\right)=1, \quad\left(\mathcal{M}_{j}\left(k_{j}\right)\right)_{11}=\left(\mathcal{M}_{j}\left(k_{j}\right)\right)_{22}
$$

which can be regarded as the renormalization-group equation describing the scaling behavior. The "renormalized" transmission coefficient $T_{j}\left(k_{j}\right)$ for $\mathrm{C}_{j}$ is given by

$$
T_{j}\left(k_{j}\right)=\frac{4}{\left|\mathcal{M}_{j}\left(k_{j}\right)\right|^{2}+2} \text {. }
$$

\section{CHAOTIC PROPAGATION, COMPLETE TRANSMISSION, AND COM- PLETE REFLECTION OF LIGHT}

In this section we study the scaling behaviors of the wave propagation by using the renormalization-group equation (14). Using analytical and numerical methods, we find two different scaling behaviors depending on $\kappa$ of the rescaled wave number $k_{j}=3^{j} \kappa$ : a) For $\kappa=\left(m / 2 \cdot 3^{q}\right) \pi$ with integers $m$ and $q$, the renormalization-group equation reduces to the logistic map describing a chaotic behavior. The renormalized transmission coefficient $T_{j}\left(k_{j}\right)$ is very sensitive to parameters of the system, and it changes drastically as the generation increases. b) For the other $\kappa$, it will be found numerically that the renormalized transmission coefficient $T_{j}\left(k_{j}\right)$ eventually flows into either $T=1$ or $T=0$ as $j \rightarrow \infty$.

A. $\kappa=\left(m / 2 \cdot 3^{q}\right) \pi$

Let us first suppose that $m$ is even, $m=2 n$. In this case, $\mathcal{T}\left(3^{j} \kappa\right)$ in (14) takes a unique value $\mathcal{T}(n \pi)$ for $j \geq q$ [26], which enables us to solve (14) analytically. By defining $\mathcal{N}_{j}=\mathcal{T}\left(3^{j+1} \kappa\right) \mathcal{M}_{j},(14)$ is recast into

$$
\mathcal{N}_{j+1}=\mathcal{N}_{j}^{2},(j \geq q), \quad \operatorname{det} \mathcal{N}_{j}=1, \quad \mathcal{N}_{j}=\mathcal{T}(n \pi) \mathcal{M}_{j}
$$

and from the relation $\left|\mathcal{N}_{j}\right|^{2}=\left|\mathcal{M}_{j}\right|^{2}$, the renormalized transmission coefficient $T_{j}\left(k_{j}\right)$ is rewritten as

$$
T_{j}=\frac{4}{\left|\mathcal{N}_{j}\right|^{2}+2}
$$



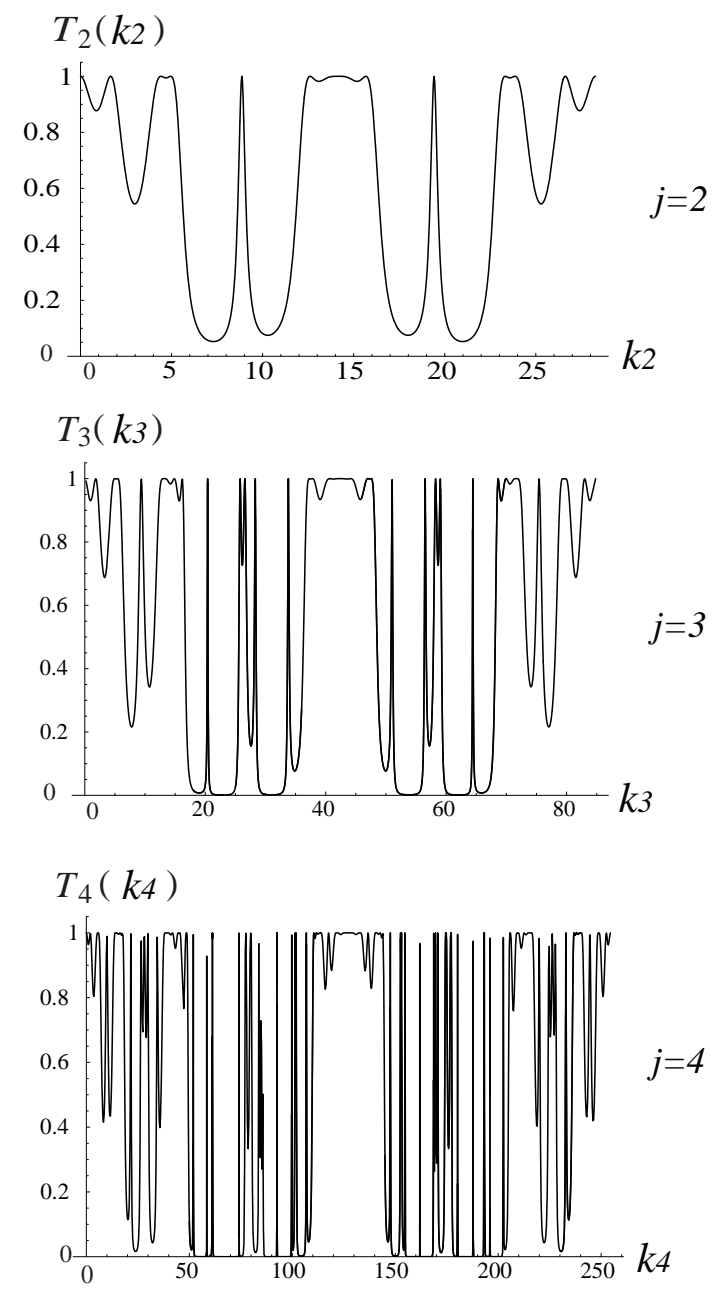

FIG. 4: Transmission coefficients $T_{j}$ as functions of the wave number $k$ for $\mathrm{C}_{2}, \mathrm{C}_{3}$, and $\mathrm{C}_{4}$ (from top to bottom) with $n_{\mathrm{A}}=2.0$. The range of the wave number $k$ for $\mathrm{C}_{j}$ is $0 \leq k \leq 3^{j} \pi$. Note that the horizontal axes are rescaled by a factor of three.

To solve (16), we rewrite $\mathcal{N}_{j}$ in terms of the Pauli matrices $\sigma_{i}(i=1,2,3)$,

$$
\mathcal{N}_{j}=\chi_{j} \mathbf{1}+\boldsymbol{\alpha}_{j} \cdot \boldsymbol{\sigma}, \quad \boldsymbol{\alpha}_{j}=\left(\alpha_{j}^{(1)}, i \alpha_{j}^{(2)}, \alpha_{j}^{(3)}\right)
$$

with real $\chi_{j}$ and $\alpha_{j}^{(i)}(i=1,2,3)$. From (16), we have

$$
\chi_{j+1}=\chi_{j}^{2}+\boldsymbol{\alpha}_{j}^{2}, \quad \boldsymbol{\alpha}_{j+1}=2 \chi_{j} \boldsymbol{\alpha}_{j}, \quad \chi_{j}^{2}-\boldsymbol{\alpha}_{j}^{2}=1, \quad(j \geq q),
$$

where $\boldsymbol{\alpha}_{j}^{2}=\left(\alpha_{j}^{(1)}\right)^{2}-\left(\alpha_{j}^{(2)}\right)^{2}+\left(\alpha_{j}^{(3)}\right)^{2}$. By eliminating $\boldsymbol{\alpha}_{j}^{2}$ in (19), the map for $\chi_{j}$ is obtained,

$$
\chi_{j+1}=2 \chi_{j}^{2}-1, \quad(j \geq q),
$$



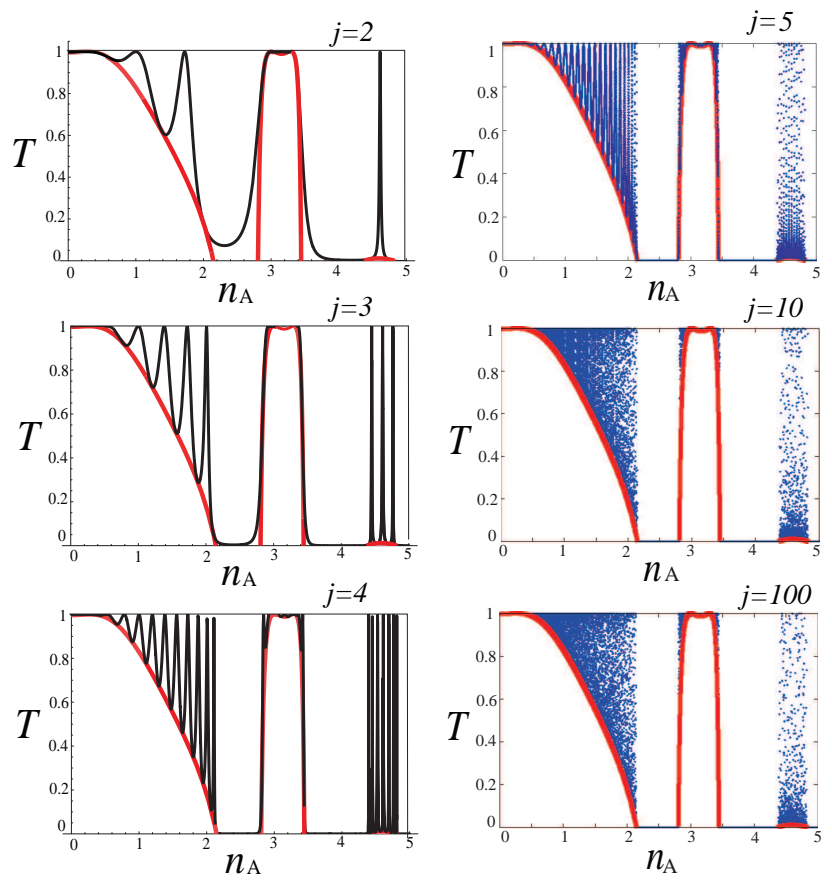

FIG. 5: (Color online) Transmission coefficients $T_{j}$ as functions of $n_{\mathrm{A}}$ for $\kappa=\pi / 3$. The generations of the left panel are $j=2, j=3$, and $j=4$. We also show transmission coefficients for $j=5$, $j=10$, and $j=100$ in the right panel. We plot $T_{\min }$ as a function of $n_{\mathrm{A}}$ by the thick red line.

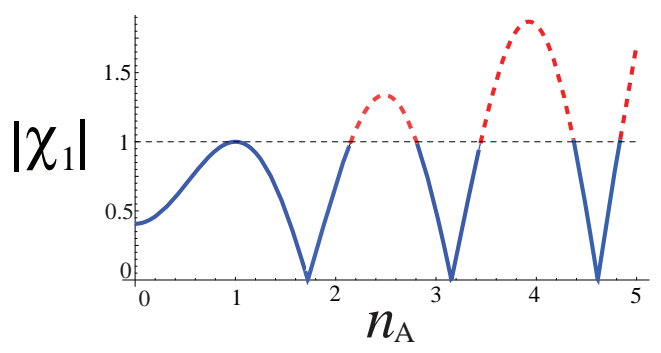

FIG. 6: (Color online) The value of $\left|\chi_{1}\right|$ as a function of $n_{\mathrm{A}}$ for $\kappa=\pi / 3$. For $n_{\mathrm{A}}$ which gives $\left|\chi_{1}\right|<1$ (solid lines), the transmission coefficients show chaotic behaviors. For $n_{\mathrm{A}}$ which gives $\left|\chi_{1}\right|>1$ (dashed lines), the transmission coefficients flow into $T=0$ for $j \rightarrow \infty$.

and in terms of the solution $\chi_{j}$ of $(\underline{201}), \boldsymbol{\alpha}_{j}$ and $T_{j}$ are represented as

$$
\boldsymbol{\alpha}_{j}=\prod_{k=q}^{j-1}\left(2 \chi_{k}\right) \boldsymbol{\alpha}_{q}, \quad T_{j}=\frac{1-\left(\lambda^{2}+\eta^{2}\right)}{1-\left(\lambda^{2}+\eta^{2}\right) \chi_{j}^{2}}, \quad(j \geq q) .
$$



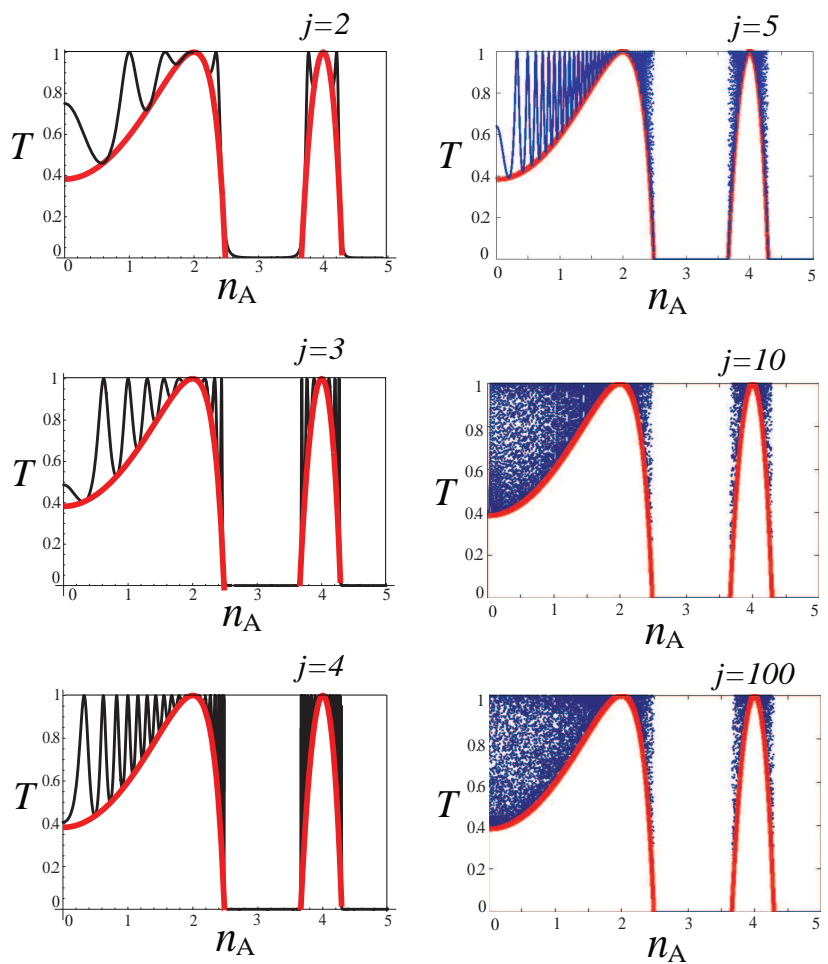

FIG. 7: (Color online) Transmission coefficients $T_{j}$ as functions of $n_{\mathrm{A}}$ for $\kappa=\pi / 2$. The generations of the left panel are $j=2, j=3$, and $j=4$. We also show transmission coefficients for $j=5$, $j=10$, and $j=100$ in the right panel. We plot $T_{\min }$ as a function of $n_{\mathrm{A}}$ by the thick red line.

Here $\lambda$ and $\eta$ are the constants of motion in (19),

$$
\frac{\alpha_{j}^{(3)}}{\alpha_{j}^{(2)}}=\frac{\alpha_{q}^{(3)}}{\alpha_{q}^{(2)}} \equiv \lambda, \quad \frac{\alpha_{j}^{(1)}}{\alpha_{j}^{(2)}}=\frac{\alpha_{q}^{(1)}}{\alpha_{q}^{(2)}} \equiv \eta, \quad(j \geq q) .
$$

For $\left|\chi_{q}\right| \leq 1$, they satisfy $\lambda^{2}+\eta^{2} \leq 1$, and for $\left|\chi_{q}\right|>1, \lambda^{2}+\eta^{2}>1$.

Equations (20) and (21) can be solved analytically. For $0 \leq\left|\chi_{q}\right|<1$, the solution is

$$
\begin{aligned}
& \chi_{j}=\cos \left[2^{j-q} \cos ^{-1} \chi_{q}\right], \quad \boldsymbol{\alpha}_{j}=\frac{\sin \left[2^{j-q} \cos ^{-1} \chi_{q}\right]}{\sin \left[\cos ^{-1} \chi_{q}\right]} \boldsymbol{\alpha}_{q} \\
& T_{j}=\frac{1-\left(\lambda^{2}+\eta^{2}\right)}{1-\left(\lambda^{2}+\eta^{2}\right) \cos ^{2}\left[2^{j-q} \cos ^{-1} \chi_{q}\right]}
\end{aligned}
$$

and for $\left|\chi_{q}\right| \geq 1$,

$$
\begin{aligned}
& \chi_{j}=\cosh \left[2^{j-q} \cosh ^{-1}\left|\chi_{q}\right|\right], \quad \boldsymbol{\alpha}_{j}=\frac{\sinh \left[2^{j-q} \cosh ^{-1}\left|\chi_{q}\right|\right]}{\sinh \left[\cosh ^{-1}\left|\chi_{q}\right|\right]} \boldsymbol{\alpha}_{q}, \\
& T_{j}=\frac{\left(\lambda^{2}+\eta^{2}\right)-1}{\left(\lambda^{2}+\eta^{2}\right) \cosh ^{2}\left[2^{j-q} \cosh ^{-1}\left|\chi_{q}\right|\right]-1} .
\end{aligned}
$$




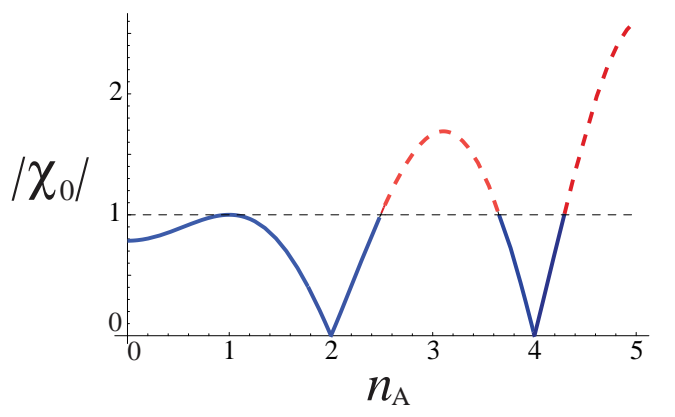

FIG. 8: (Color online) The value of $\left|\chi_{0}\right|$ as a function of $n_{\mathrm{A}}$ for $\kappa=\pi / 2$. For $n_{\mathrm{A}}$ which gives $\left|\chi_{0}\right|<1$ (solid lines), the transmission coefficients show chaotic behaviors. For $n_{\mathrm{A}}$ which gives $\left|\chi_{0}\right|>1$ (dashed lines), the transmission coefficients flow into $T=0$ for $j \rightarrow \infty$.

For $\left|\chi_{q}\right|<1$, the transmission coefficient $T_{j}$ shows sensitive dependence on parameters of the system. To illustrate this, we show the transmission coefficients for $q=1$ and $n=1$ case, (namely $\kappa=\pi / 3$ ), as functions of $n_{\mathrm{A}}$ in Fig.5. In this case, $\chi_{q=1}$ is given by

$$
\chi_{1}=-\frac{1}{2} \cos \left(\frac{2 n_{\mathrm{A}} \pi}{3}\right)+\frac{\sqrt{3}}{4}\left(n_{\mathrm{A}}+\frac{1}{n_{\mathrm{A}}}\right) \sin \left(\frac{2 n_{\mathrm{A}} \pi}{3}\right),
$$

and the region of $n_{\mathrm{A}}$ with $\left|\chi_{1}\right|<1$ is shown in Fig, [6. It is found clearly that the transmission coefficients are very sensitive to $n_{\mathrm{A}}$ in the region where $\left|\chi_{1}\right|<1$.

This sensitivity to parameters of the system can be properly understood by introducing a new variable $X_{j}=1-\chi_{j}^{2}$. In terms of the new variable, (20) reduces to the logistic map with $r=4$,

$$
X_{j+1}=r X_{j}\left(1-X_{j}\right)
$$

which has been studied extensively in the context of chaos [20, 21, 22, 23]. The logistic map with $r=4$ in the interval $0<X<1$ is known to be very sensitive to the initial condition of the system, which implies that $T_{j}$ for $\left|\chi_{q}\right|<1$ also has the same chaotic property.

A similar chaotic behavior of the transmission coefficient also appears for odd $m=2 n+1$,

$$
\kappa=\frac{2 n+1}{2 \cdot 3^{q}} \pi
$$

The matrix $\mathcal{T}\left(3^{j} \kappa\right)$ in (14) is now $(-1)^{j-q} \mathcal{T}((n+1 / 2) \pi)$ for $j \geq q$, so $\mathcal{N}_{j}\left(\equiv \mathcal{T}\left(3^{j+1} \kappa\right) \mathcal{M}_{j}\right)$ becomes $\mathcal{N}_{j}=(-1)^{j+1-q} \mathcal{T}((n+1 / 2) \pi) \mathcal{M}_{j}$. For this $\mathcal{N}_{j}$, we have the same equation as (16)),

$$
\mathcal{N}_{j+1}=\mathcal{N}_{j}^{2}, \quad \operatorname{det} \mathcal{N}_{j}=1
$$


and the same expression of the transmission coefficient as (17),

$$
T_{j}=\frac{4}{\left|\mathcal{N}_{j}\right|^{2}+2}
$$

By using $\chi_{j}, \boldsymbol{\alpha}_{j}, \lambda$ and $\eta$ defined in (18) and (22), the same solutions (23) and (24) are obtained. Thus we have the same class of chaotic behavior. As an example, we show the transmission coefficients $T_{j}$ for $\kappa=\pi / 2(n=q=0$ in (27) $)$ as functions of $n_{\mathrm{A}}$ in Fig:7, For $\kappa=\pi / 2, \chi_{q=0}$ is given by

$$
\chi_{0}=\frac{1}{2}\left(n_{\mathrm{A}}+\frac{1}{n_{\mathrm{A}}}\right) \sin \left(\frac{n_{\mathrm{A}} \pi}{2}\right) .
$$

and the region with $\left|\chi_{0}\right|<1$ is shown in Fig.8. We find again that $T_{j}$ is very sensitive to $n_{\mathrm{A}}$ in the region with $\left|\chi_{0}\right|<1$.

In the chaotic region, $\left|\chi_{q}\right|<1$, the transmission coefficient $T_{j}$ has the lower bound $T_{\min }$

$$
T_{\min }=1-\left(\lambda^{2}+\eta^{2}\right)
$$

For $\kappa=\pi / 3, \lambda$ and $\eta$ are given by

$$
\lambda=0, \quad \eta=\frac{-\sqrt{3}\left(n_{\mathrm{A}}^{2}-n_{\mathrm{A}}^{-2}\right) \sin ^{2}\left(\pi n_{\mathrm{A}} / 3\right)+\left(n_{\mathrm{A}}-n_{\mathrm{A}}^{-1}\right) \sin \left(2 \pi n_{\mathrm{A}} / 3\right)}{\sqrt{3}\left(n_{\mathrm{A}}+n_{\mathrm{A}}^{-1}\right)^{2} \sin ^{2}\left(\pi n_{\mathrm{A}} / 3\right)-\left(n_{\mathrm{A}}+n_{\mathrm{A}}^{-1}\right) \sin \left(2 \pi n_{\mathrm{A}} / 3\right)-2 \sqrt{3}},
$$

and for $\kappa=\pi / 2$,

$$
\lambda=\frac{1}{2}\left(n_{\mathrm{A}}-\frac{1}{n_{\mathrm{A}}}\right) \tan \left(\frac{\pi n_{\mathrm{A}}}{2}\right), \quad \eta=0 .
$$

The resultant $T_{\min }$ 's as functions of $n_{\mathrm{A}}$ are also depicted in Figs 5 and 7 , respectively.

From the analytic solution (24), we notice that outside the chaotic region, $\left|\chi_{q}\right| \geq 1, T_{j}$ decreases monotonically as the generation $j$ increases. Thus we have complete reflection $T_{j}=0$ for $j \rightarrow \infty$.

\section{B. Other $\kappa$}

For $\kappa \neq\left(m / 2 \cdot 3^{q}\right) \pi$, we numerically find that the transmission coefficient $T_{j}$ flows into either $T=1$ or $T=0$ as the generation $j$ increases. A typical example of our numerical results is shown in Fig.9. It is also found that if complete transmission of light occurs at a certain generation, light always transmits completely in the following generations. To see 

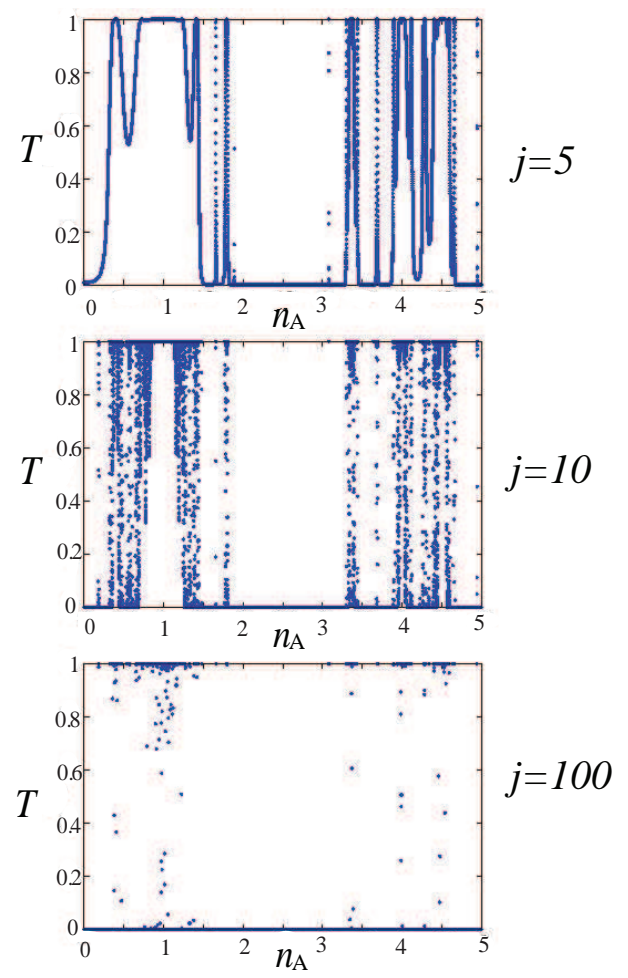

FIG. 9: (Color online) Transmission coefficients $T_{j}$ as functions of $n_{\mathrm{A}}$ for $\kappa=\pi / 4$. The generations are $j=5, j=10$, and $j=100$ (from top to bottom).

this, let us use the component representation of $\mathcal{M}_{j}\left(k_{j}\right)$ as (12),

$$
\mathcal{M}_{j}\left(k_{j}\right)=\left(\begin{array}{cc}
a_{j} & b_{j} \\
c_{j} & a_{j}
\end{array}\right)
$$

which satisfies $a_{j}^{2}-b_{j} c_{j}=1$. If $T_{j}=1,2 a_{j}^{2}+b_{j}^{2}+c_{j}^{2}=2$ from (15), thus $\mathcal{M}_{j}$ can be parameterized as

$$
\mathcal{M}_{j}\left(k_{j}\right)=\left(\begin{array}{cc}
\cos \delta_{j} & -\sin \delta_{j} \\
\sin \delta_{j} & \cos \delta_{j}
\end{array}\right), \quad a_{j}=\cos \delta_{j}, \quad b_{j}=-c_{j}=-\sin \delta_{j}
$$

Substituting this into (14) yields

$$
\mathcal{M}_{j+1}\left(k_{j+1}\right)=\left(\begin{array}{cc}
\cos \left(2 \delta_{j}+k_{j}\right) & -\sin \left(2 \delta_{j}+k_{j}\right) \\
\sin \left(2 \delta_{j}+k_{j}\right) & \cos \left(2 \delta_{j}+k_{j}\right)
\end{array}\right),
$$

which implies $T_{j+1}=1$. 

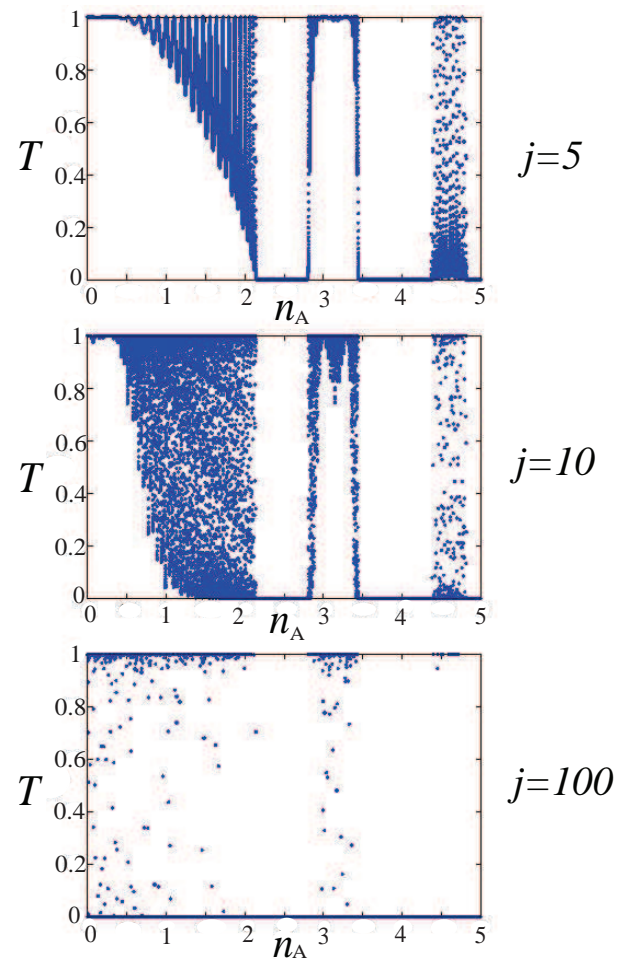

FIG. 10: (Color online) Transmission coefficients $T_{j}$ as functions of $n_{\mathrm{A}}$ for $\kappa=\pi / 3+10^{-4}$. The generations are $j=5, j=10$, and $j=100$ (from top to bottom).

When $\kappa$ is near $\kappa=\left(m / 2 \cdot 3^{q}\right) \pi$, the transmission coefficient is found to show transition from the chaotic behavior presented in the previous subsection: The chaotic behavior appears in first few generations, but finally the transmission coefficient flows into either complete transmission or complete reflection. For example, the transmission coefficient $T_{j}$ for $\kappa=$ $\pi / 3+10^{-4}$ is shown in Fig.10. In the fifth generation $(j=5)$, the transmission coefficient shows a chaotic behavior indistinguishable from that for $\kappa=\pi / 3$ (Fig 5 ), however, in the tenth generation $(j=10)$, it shows a different behavior from that for $\kappa=\pi / 3$, then finally in the hundredth generation $(j=100)$, it flows into either $T=1$ or $T=0$.

\section{Optical experiments}

The chaotic behaviors of the transmission coefficients presented in Sec $\amalg$ II can be observed experimentally. As was shown in Figs.5 and 7 , the chaotic characteristics in the propagation are already evident in the first few generations of the Cantor multilayers, where 
the transmission coefficients oscillate rapidly as functions of $n_{\mathrm{A}}$. (This oscillation reflects the stretching and the folding process of the logistic map.) Therefore, the finite generations of the Cantor multilayers are sufficient to observe the chaotic behaviors. Experimentally, the finite generations of $\mathrm{C}_{j}$ can be fabricated by using the vacuum deposition on a glass substrate [5, 16, 17]. In a similar manner as the Fibonacci multilayers [16, 17], the Cantor multilayers are prepared from $\mathrm{SiO}_{2}$ and $\mathrm{TiO}_{2}$ films. $\mathrm{Na}_{3} \mathrm{AlF}_{6}$ and $\mathrm{ZnS}$ are also utilized for

the fabrication of $\mathrm{C}_{j}[5]$. Although the wave number $k$ needs to satisfy the condition $k=3^{j} \kappa$ with $\kappa=\left(\mathrm{m} / 2 \cdot 3^{q}\right) \pi$ ( $\mathrm{m}$ and $q$ are integers) in order to observe the chaotic behaviors on the $j$ th generation of the Cantor multilayers, this condition can be met by using tunable lasers.

From the argument in Sec $\underline{\text { IIA }}$, the indices of the refraction of A and B should be set in the region with $0 \leq\left|\chi_{q}\right|<1$. Suppose that the index of refraction of B is 1 , then $\chi_{q}$ is given by (see Eq.(18))

$$
\chi_{q}=\frac{1}{2} \operatorname{Tr}\left(\mathcal{T}\left(3^{q+1} \kappa\right) \mathcal{M}_{q}\right)
$$

where $\mathcal{M}_{q}\left(\equiv \mathcal{M}_{q}\left(k_{q}\right)\right)$ is the solution of Eq.(14). For example, in the case of $\kappa=\pi / 3$ $(\kappa=\pi / 2), \chi_{q=1}\left(\chi_{q=0}\right)$ as a function of the index of the refraction of $\mathrm{A}, n_{\mathrm{A}}$, is given by Eq.(25) (Eq.(30) ). As was illustrated in Fig.6 (Fig, (8), the condition $0 \leq\left|\chi_{q}\right|<1$ can be met in a broad region of $n_{\mathrm{A}}$ without fine tuning. Therefore, the detection of the chaotic behaviors is feasible for the current optical experiments.

The most impressive chaotic behavior is obtained if $n_{\mathrm{A}}$ satisfies $\left|\chi_{q}\right| \sim 1$. Near $n_{\mathrm{A}}$ satisfying $\left|\chi_{q}\right|=1$, all values of $T$ appear in a very narrow region of $n_{\mathrm{A}}$. In particular, complete transmission and (almost) complete reflection are nearby in the narrow region. By controlling $n_{\mathrm{A}}$, the Cantor multilayers with $\left|\chi_{q}\right| \sim 1$ could be used as fast switching devices.

\section{LOCAL SCALING BEHAVIOR OF TRANSMISSION COEFFICIENTS NEAR COMPLETE TRANSMISSION}

In the previous section, we found two classes of behaviors of the transmission coefficients where they remain finite: chaotic behaviors and complete transmission. In this section, we focus on a behavior of the transmission coefficient near complete transmission. As we showed in the previous section, if we have complete transmission of light at a certain generation, light always transmits completely after the generation. Thus we can consider that complete 
transmission is a fixed point of the renormalization-group equation (14). According to the renormalization-group theory, the existence of a fixed point implies that scalings are found around it [24], which turns out to be also true in our problem. In the following, on the basis of the renormalization-group equation, it will be shown that if complete transmission is achieved at a wave number $\kappa^{*}$ with a rational $\kappa^{*} / \pi$, then the transmission coefficient $T_{j}$ around the wave number exhibits a local scaling behavior which is distinct from the global scaling illustrated in Fig,4. Moreover, we will present the analytic expression of the scaling function.

In order to analyze the scaling behavior of $T_{j}$ near $T=1$, it is convenient to introduce new variables $x_{j} \equiv a_{j}\left(k_{j}\right), y_{j} \equiv\left(b_{j}\left(k_{j}\right)-c_{j}\left(k_{j}\right)\right) / 2$ and $z_{j} \equiv\left(b_{j}\left(k_{j}\right)+c_{j}\left(k_{j}\right)\right) / 2$ for the matrix elements of $\mathcal{M}_{j}\left(k_{j}\right)$,

$$
\mathcal{M}_{j}\left(k_{j}\right)=\left(\begin{array}{cc}
a_{j}\left(k_{j}\right) & b_{j}\left(k_{j}\right) \\
c_{j}\left(k_{j}\right) & a_{j}\left(k_{j}\right)
\end{array}\right), \quad k_{j}=3^{j} \kappa .
$$

Since $a_{j}\left(k_{j}\right), b_{j}\left(k_{j}\right)$ and $c_{j}\left(k_{j}\right)$ satisfy $a_{j}\left(k_{j}\right)^{2}-b_{j}\left(k_{j}\right) c_{j}\left(k_{j}\right)=1$, the new variables $\left(x_{j}, y_{j}, z_{j}\right)$ are constrained on the manifold $x_{j}^{2}+y_{j}^{2}=1+z_{j}^{2}$ shown in Fig 11 . By rewriting $x_{j}$ and $y_{j}$ as

$$
x_{j}=\sqrt{z_{j}^{2}+1} \cos \varphi_{j}, \quad y_{j}=\sqrt{z_{j}^{2}+1} \sin \varphi_{j},
$$

the map (14) is recast into

$$
\begin{aligned}
z_{j+1} & =2 z_{j} \sqrt{z_{j}^{2}+1} \cos \left(\varphi_{j}-3^{j} \kappa\right), \\
\sqrt{z_{j+1}^{2}+1} e^{i \varphi_{j+1}} & =\left(z_{j}^{2}+1\right) e^{i\left(2 \varphi_{j}-3^{j} \kappa\right)}+z_{j}^{2} e^{i 3^{j} \kappa}
\end{aligned}
$$

and the transmission coefficient $T_{j}$ in (15) is rewritten as

$$
T_{j}=\frac{1}{x_{j}^{2}+y_{j}^{2}}=\frac{1}{1+z_{j}^{2}} .
$$

The complete transmission $T_{j}=1$ is achieved when $z_{j}=0$. From (40), it can be found that this occurs either if 1$) z_{l}=0$ with $z_{l-1} \neq 0$ for an integer $l(1 \leq l \leq j)$, or 2$) z_{0}=0$.

First consider the case 1 ) in detail. Suppose that $z_{l}=0$ with $z_{l-1} \neq 0$ is realized for $\kappa=\kappa_{l}^{*}$. Then from the first equation of (40), it is found that the case 1) is possible only if $\cos \left(\varphi_{l-1}-3^{l-1} \kappa_{l}^{*}\right)=0$. Thus $\varphi_{l-1}$ is given by

$$
\varphi_{l-1}=3^{l-1} \kappa_{l}^{*}+\frac{2 n+1}{2} \pi,
$$




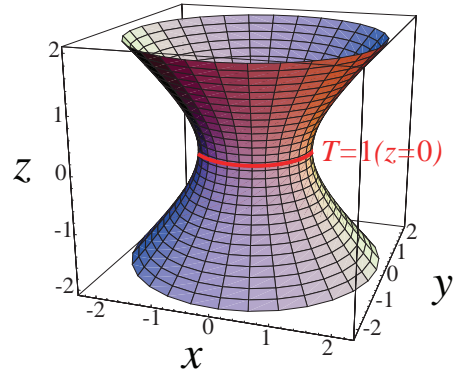

FIG. 11: (Color online) Orbits of (14) are constrained on the manifold $x^{2}+y^{2}=z^{2}+1$. Complete transmission $T=1$ corresponds to $z=0$.

with an integer $n$. Then using (40) with $j=l-1$ and $z_{l}=0$, we have

$$
\begin{aligned}
e^{i \varphi_{l}} & =\left(z_{l-1}^{2}+1\right) e^{i\left(2 \varphi_{l-1}-3^{l-1} \kappa_{l}^{*}\right)}+z_{l-1}^{2} e^{i 3^{l-1} \kappa_{l}^{*}} \\
& =\left(z_{l-1}^{2}+1\right) e^{i\left(3^{l-1} \kappa_{l}^{*}+(2 n+1) \pi\right)}+z_{l-1}^{2} e^{i 3^{l-1} \kappa_{l}^{*}} \\
& =-e^{i 3^{l-1} \kappa_{l}^{*}} .
\end{aligned}
$$

Hence $\varphi_{l}$ is obtained as

$$
\varphi_{l}=3^{l-1} \kappa_{l}^{*}+\pi, \quad(\bmod 2 \pi) .
$$

To determine $\varphi_{j}$ for $j \geq l$, we use the second equation of (40). Since $z_{j}=0$ for $j \geq l$, the second equation of (40) becomes

$$
e^{i \varphi_{j+1}}=e^{i\left(2 \varphi_{j}-3^{j} \kappa_{l}^{*}\right)} \quad(j \geq l)
$$

which determines $\varphi_{j}$ for $j \geq l$ completely as,

$$
\varphi_{j}=-\left(3^{j}-2^{j-l} 3^{l}\right) \kappa_{l}^{*}+2^{j-l} \varphi_{l} \quad(j \geq l ; \bmod 2 \pi)
$$

where $\varphi_{l}$ is given by (44).

In a similar manner, we can also solve $\varphi_{j}$ in the case 2). Suppose that $z_{0}=0$ for $\kappa=\kappa_{0}^{*}$. From the following explicit form of $\mathcal{M}_{0}$,

$$
\mathcal{M}_{0}\left(k_{0}\right)=\mathcal{T}_{\mathrm{BA}} \mathcal{T}_{\mathrm{A}}\left(n_{\mathrm{A}} k_{0}\right) \mathcal{T}_{\mathrm{AB}}=\left(\begin{array}{cc}
\cos n_{\mathrm{A}} \kappa & -\left(1 / n_{\mathrm{A}}\right) \sin n_{\mathrm{A}} \kappa \\
n_{\mathrm{A}} \sin n_{\mathrm{A}} \kappa & \cos n_{\mathrm{A}} \kappa
\end{array}\right)
$$

we obtain that $z_{0}=\left(n_{\mathrm{A}}-1 / n_{\mathrm{A}}\right) \sin \left(n_{\mathrm{A}} \kappa\right) / 2$. Therefore, $\kappa_{0}^{*}$ is given by $\kappa_{0}^{*}=m \pi / n_{\mathrm{A}}$ with an integer $m$. (Note that the indices of the refraction of A and B are different from each other, 
i.e. $n_{\mathrm{A}} \neq 1$.) Substituting this into (47), we find that $x_{0}=\cos m \pi$ and $y_{0}=0$. Thus $\varphi_{0}$ is given by

$$
\varphi_{0}=m \pi, \quad(\bmod 2 \pi) .
$$

Since $z_{j}=0$ for $j \geq 0$, we have

$$
e^{i \varphi_{j+1}}=e^{i\left(2 \varphi_{j}-3^{j} \kappa_{0}^{*}\right)} \quad(j \geq 0)
$$

from Eq.(40), which determines $\varphi_{j}$ as

$$
\varphi_{j}=-\left(3^{j}-2^{j}\right) \kappa_{0}^{*}+2^{j} \varphi_{0}, \quad(\bmod 2 \pi)
$$

with $\varphi_{0}=m \pi$.

Now we study behavior of the transmission coefficient $T_{j}$ near $T=1$. For $\kappa$ near $\kappa_{l}^{*}, z_{l}$ becomes nonzero but remains small, so we can neglect higher order terms of $z_{i}^{2}(i \geq l)$ in the map (40). Up to the next leading order, the map (40) is approximated by

$$
z_{j+1}=2 z_{j} \cos \left(\varphi_{j}-3^{j} \kappa\right), \quad e^{i \varphi_{j+1}}=e^{i\left(2 \varphi_{j}-3^{j} \kappa\right)}
$$

The latter equation in (51) can be solved easily,

$$
\varphi_{j}=-\left(3^{j}-2^{j-l} 3^{l}\right) \kappa+2^{j-l} \varphi_{l}, \quad(\bmod 2 \pi)
$$

where $\varphi_{l}$ is given by

$$
\varphi_{l}=\left\{\begin{array}{ll}
3^{l-1} \kappa_{l}^{*}+\pi+O\left(z_{l}\right), & \text { for } l \neq 0 \\
m \pi+O\left(z_{0}\right), & \text { for } l=0
\end{array} . \quad(\bmod 2 \pi) .\right.
$$

Here we have determined $\varphi_{l}$ in a similar manner as (44) and (48), but $O\left(z_{l}\right)$ corrections appear since $z_{l} \neq 0$ near $\kappa_{l}^{*}$. Substituting this into the first equation in (51), we obtain

$$
z_{j+1}=2 z_{j} \cos \gamma_{j}^{(l)}(\kappa)
$$

where $\gamma_{j}^{(l)}(\kappa)$ denotes

$$
\gamma_{j}^{(l)}(\kappa)=\left\{\begin{array}{ll}
\left(2 \cdot 3^{j}-2^{j-l} 3^{l}\right) \kappa-2^{j-l}\left(3^{l-1} \kappa_{l}^{*}+\pi+O\left(z_{l}\right)\right), & \text { for } l \neq 0 \\
\left(2 \cdot 3^{j}-2^{j}\right) \kappa-2^{j}\left(m \pi+O\left(z_{0}\right)\right), & \text { for } l=0
\end{array} \quad(\bmod \pi) .\right.
$$


Therefore, for $\kappa$ near $\kappa_{l}^{*}, z_{j}$ is given by

$$
z_{j}=z_{l} \prod_{i=l}^{j-1}\left(2 \cos \gamma_{i}^{(l)}(\kappa)\right)
$$

Using this, we obtain the following transmission coefficient $T_{j}$ near $\kappa_{l}^{*}$,

$$
T_{j}=1-z_{l}^{2} \prod_{i=l}^{j-1}\left(2 \cos \gamma_{i}^{(l)}(\kappa)\right)^{2} .
$$

As is proved in Appendix $\mathrm{A}$, we can show that if $\kappa_{l}^{*} / \pi$ is a rational number $s / t$ with coprime integers $s$ and $t$, the phase $\gamma_{i}^{(l)}\left(\kappa_{l}^{*}\right)$ becomes periodic for a sufficiently large $i$,

$$
\gamma_{i+p}^{(l)}\left(\kappa_{l}^{*}\right)=\gamma_{i}^{(l)}\left(\kappa_{l}^{*}\right), \quad(i \geq r ; \bmod \pi)
$$

where the minimal period $p$ is given by a divisor of $\varphi(t)$. (Here $\varphi(x)$ is the Euler's totient function[25].) Using this, we obtain a local scaling behavior near complete transmission. To see this, rewrite Eq.(57) by using $\kappa=\kappa_{l}^{*}+\theta(\theta \ll 1)$,

$$
T_{j}(\theta)=1-z_{l}^{2} \prod_{i=l}^{j-1}\left(2 \cos \left[\gamma_{i}^{(l)}\left(\kappa_{l}^{*}\right)+\gamma_{i}^{(l)^{\prime}}\left(\kappa_{l}^{*}\right) \theta\right]\right)^{2} .
$$

If $\kappa_{l}^{*} / \pi$ is a rational number $s / t$, then from the relation (58), the product in the right-hand side of (59) for $j=p n+m-1(m=1,2, \cdots, p)$ is rewritten as

$$
\begin{aligned}
& \prod_{i=l}^{p n+m-2}\left(2 \cos \left[\gamma_{i}^{(l)}\left(\kappa_{l}^{*}\right)+\gamma_{i}^{(l)^{\prime}}\left(\kappa_{l}^{*}\right) \theta\right]\right)^{2} \\
= & \prod_{i=p(n-1)+m-1}^{p n+m-2}\left(2 \cos \left[\gamma_{i}^{(l)}\left(\kappa_{l}^{*}\right)+\gamma_{i}^{(l)^{\prime}}\left(\kappa_{l}^{*}\right) \theta\right]\right)^{2} \prod_{i=l}^{p(n-1)+m-2}\left(2 \cos \left[\gamma_{i}^{(l)}\left(\kappa_{l}^{*}\right)+\gamma_{i}^{(l)^{\prime}}\left(\kappa_{l}^{*}\right) \theta\right]\right)^{2} \\
= & \prod_{\alpha=0}^{p-1}\left(2 \cos \left[\gamma_{p(n-1)+m-1+\alpha}^{(l)}\left(\kappa_{l}^{*}\right)+\gamma_{p(n-1)+m-1+\alpha}^{(l)}\left(\kappa_{l}^{*}\right) \theta\right]\right)^{2} \prod_{i=l}^{p(n-1)+m-2}\left(2 \cos \left[\gamma_{i}^{(l)}\left(\kappa_{l}^{*}\right)+\gamma_{i}^{(l)^{\prime}}\left(\kappa_{l}^{*}\right) \theta\right]\right)^{2} \\
= & \prod_{\alpha=0}^{p-1}\left(2 \cos \left[\gamma_{p n_{0}(m)+m-1+\alpha}^{(l)}\left(\kappa_{l}^{*}\right)+\gamma_{p(n-1)+m-1+\alpha}^{(l)^{\prime}}\left(\kappa_{l}^{*}\right) \theta\right]\right)^{2} \prod_{i=l}^{p(n-1)+m-2}\left(2 \cos \left[\gamma_{i}^{(l)}\left(\kappa_{l}^{*}\right)+\gamma_{i}^{(l)^{\prime}}\left(\kappa_{l}^{*}\right) \theta\right]\right)^{2},
\end{aligned}
$$

where $n_{0}(m)$ is the minimal integer satisfying $p n_{0}+m-1 \geq r$. Therefore, we have $1-T_{p n+m-1}(\theta)=\prod_{\alpha=0}^{p-1}\left(2 \cos \left[\gamma_{p n_{0}(m)+m-1+\alpha}^{(l)}\left(\kappa_{l}^{*}\right)+\gamma_{p(n-1)+m-1+\alpha}^{(l)^{\prime}}\left(\kappa_{l}^{*}\right) \theta\right]\right)^{2}\left(1-T_{p(n-1)+m-1}(\theta)\right)$. 


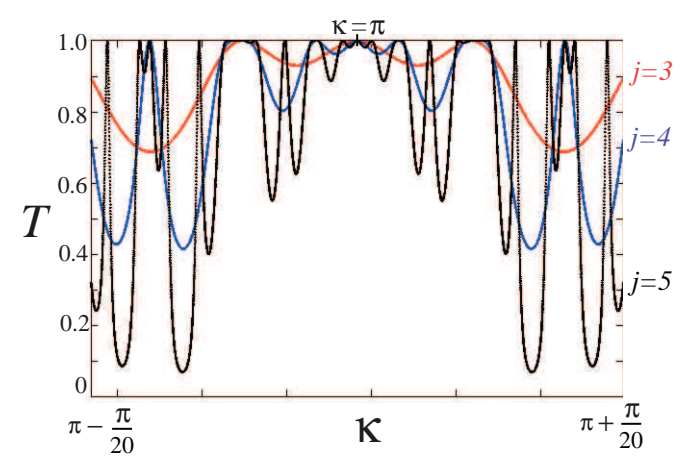

FIG. 12: (Color online) Transmission coefficients $T_{j}$ as functions of $\kappa$ for $n_{\mathrm{A}}=2.0$ around $\kappa_{0}^{*}=\pi$. The generations are $j=3, j=4$, and $j=5$.

From Eq. (55), $\gamma_{p(n-1)+m-1+\alpha}^{(l)^{\prime}}\left(\kappa_{l}^{*}\right)$ behaves as

$$
\gamma_{p(n-1)+m-1+\alpha}^{(l)^{\prime}}\left(\kappa_{l}^{*}\right) \sim 2 \cdot 3^{p(n-1)+m-1+\alpha},
$$

for $n \gg 1$. Thus defining $f^{(m)}(\theta)$ as

$$
f^{(m)}(\theta)=\prod_{\alpha=0}^{p-1}\left(2 \cos \left[\gamma_{p n_{0}(m)+m-1+\alpha}^{(l)}\left(\kappa_{l}^{*}\right)+2 \cdot 3^{\alpha} \theta\right]\right)^{2},
$$

we obtain

$$
1-T_{p n+m-1}(\theta)=f^{(m)}\left(3^{p(n-1)+m-1} \theta\right)\left(1-T_{p(n-1)+m-1}(\theta)\right) \quad(n \gg 1) .
$$

By renormalizing $T_{p n+m-1}(\theta)$ as

$$
\hat{T}_{p n+m-1}(\theta) \equiv T_{p n+m-1}\left(\theta / 3^{p(n-1)+m-1}\right)
$$

Eq.(64) is rewritten as

$$
1-\hat{T}_{p n+m-1}(\theta)=f^{(m)}(\theta)\left(1-\hat{T}_{p(n-1)+m-1}\left(\theta / 3^{p}\right)\right)
$$

This equation clearly indicates that the ratio $\left(1-\hat{T}_{p n+m-1}(\theta)\right) /\left(1-\hat{T}_{p(n-1)+m-1}\left(\theta / 3^{p}\right)\right)$ does not depend on the generation of Cantor sequences and it has a scaling behavior with the scaling function $f^{(m)}(\theta)$.

To illustrate the local scaling behavior of $T_{j}$ near $T=1$ obtained above, we compare our formula (66) with numerical results for various $\kappa_{l}^{*}$. In Figs 12 and 13, we show the 
(a)
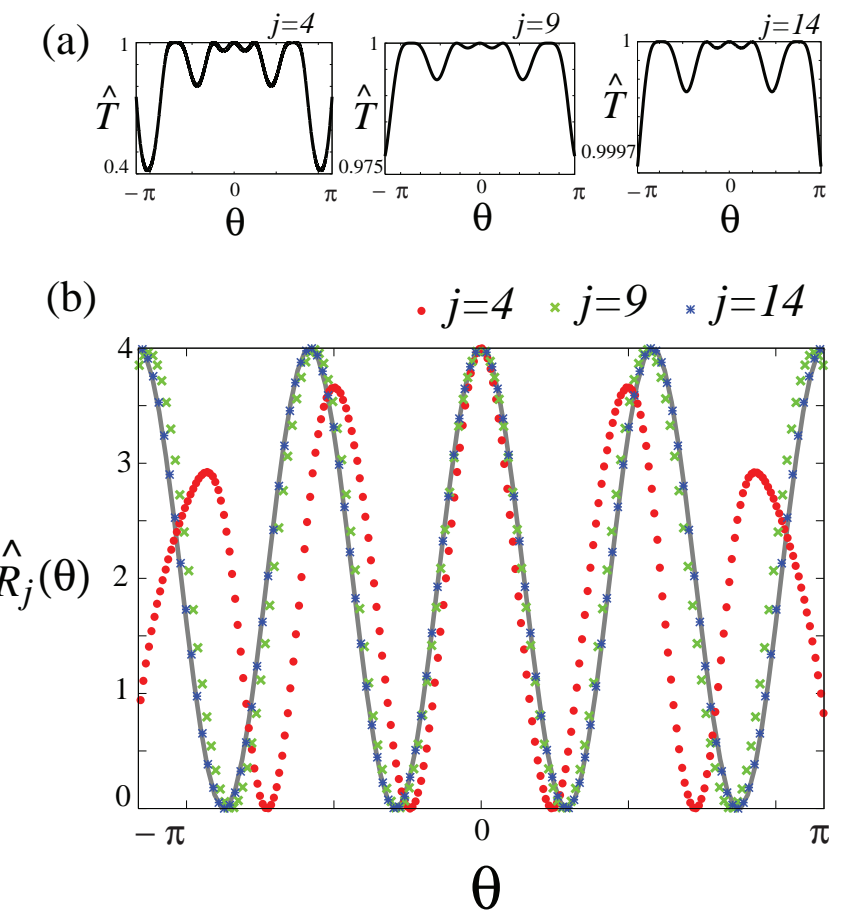

FIG. 13: (Color online) (a) Transmission coefficients $\hat{T}_{j}$ as functions of $\theta$ for $n_{\mathrm{A}}=2.0$ around $\kappa_{0}^{*}=$ $\pi$. The generations are $j=4, j=9$, and $j=14$. (b) The ratio $\hat{R}_{j}(\theta)=\left(1-\hat{T}_{j}(\theta)\right) /\left(1-\hat{T}_{j-1}(\theta / 3)\right)$ for $n_{\mathrm{A}}=2.0$ around $\kappa_{0}^{*}=\pi$. The generations are $j=4, j=9$, and $j=14$. The scaling function $f^{(1)}(\theta)$ is also plotted by the line.

transmission coefficient $T_{j}$ for $n_{\mathrm{A}}=2$ and $\kappa_{0}^{*}=\pi$. The transmission coefficients $T_{j}$ as functions of $\kappa$ are presented in Fig.12 and the renormalized one $\hat{T}_{j}(\theta)$ is in Fig 13 . In this case, the period $p$ in (58) is $p=\varphi(1)=1$, and the scaling function $f^{(1)}(\theta)$ is

$$
f^{(1)}(\theta)=4 \cos ^{2}(2 \theta)
$$

For $j \geq 9$ we find an excellent agreement between our formula (66) and numerical data in Fig.13.

In Figs 14, 16, we also present local scaling behaviors for $n_{\mathrm{A}}=t$ and $\kappa_{0}^{*}=\pi / t$ with integers $t=2,3,5$. For $t=2,3,5$, the period $p$ is given by $p=1,2,4$, respectively. The scaling functions $f^{(m)}(\theta)$ are given by

$$
f^{(1)}(\theta)=4 \cos ^{2}(2 \theta)
$$



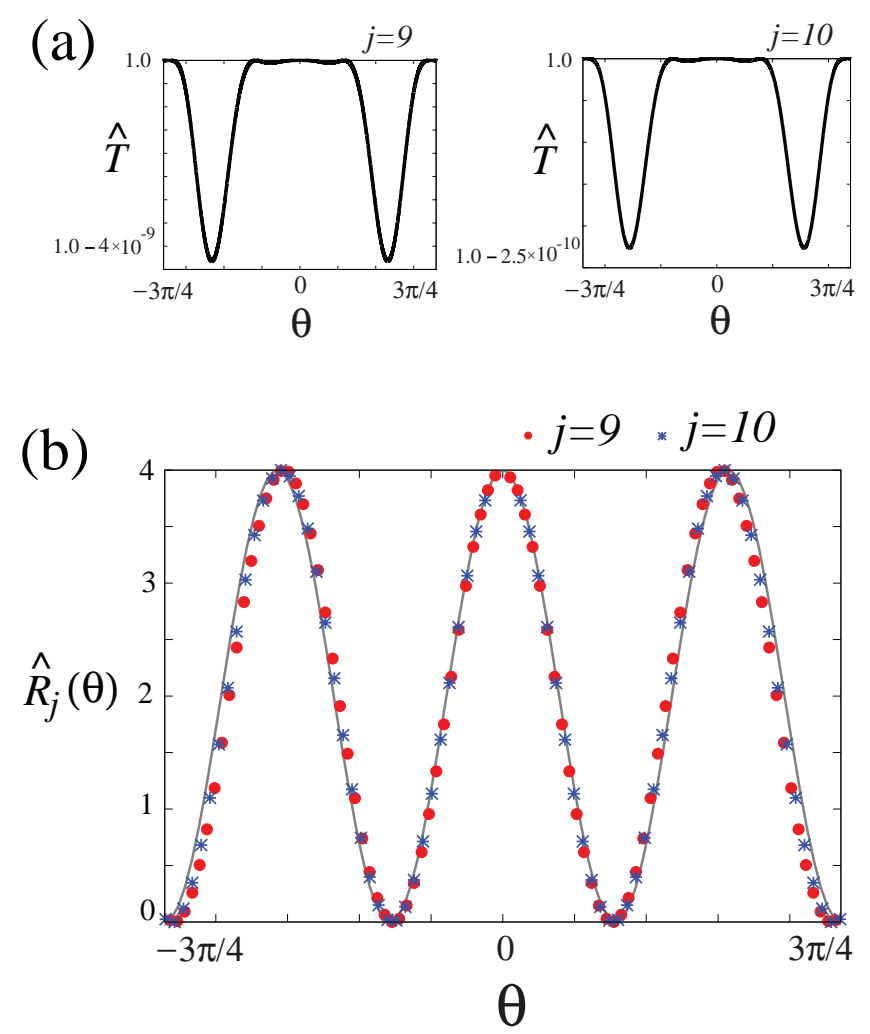

FIG. 14: (Color online) (a) Transmission coefficients $\hat{T}_{j}$ as functions of $\theta$ for $n_{\mathrm{A}}=2.0$ around $\kappa_{0}^{*}=\pi / 2$. The generations are $j=9$ and $j=10$. (b) The ratio $\hat{R}_{j}(\theta)=\left(1-\hat{T}_{j}(\theta)\right) /\left(1-\hat{T}_{j-1}(\theta / 3)\right)$ for $n_{\mathrm{A}}=2.0$ around $\kappa_{0}^{*}=\pi / 2$. The generations are $j=9$ and $j=10$. The scaling function $f^{(1)}(\theta)$ is also plotted by the line.

for $t=2$, and

$$
\begin{aligned}
& f^{(1)}(\theta)=16 \cos ^{2}(\pi / 3+6 \theta) \cos ^{2}(2 \pi / 3+2 \theta), \\
& f^{(2)}(\theta)=16 \cos ^{2}(2 \pi / 3+6 \theta) \cos ^{2}(\pi / 3+2 \theta)
\end{aligned}
$$

for $t=3$, and

$$
\begin{aligned}
& f^{(1)}(\theta)=256 \cos ^{2}(\pi / 5+54 \theta) \cos ^{2}(4 \pi / 5+18 \theta) \cos ^{2}(4 \pi / 5+6 \theta) \cos ^{2}(\pi / 5+2 \theta), \\
& f^{(2)}(\theta)=256 \cos ^{2}(\pi / 5+54 \theta) \cos ^{2}(\pi / 5+18 \theta) \cos ^{2}(4 \pi / 5+6 \theta) \cos ^{2}(4 \pi / 5+2 \theta), \\
& f^{(3)}(\theta)=256 \cos ^{2}(4 \pi / 5+54 \theta) \cos ^{2}(\pi / 5+18 \theta) \cos ^{2}(\pi / 5+6 \theta) \cos ^{2}(4 \pi / 5+2 \theta), \\
& f^{(4)}(\theta)=256 \cos ^{2}(4 \pi / 5+54 \theta) \cos ^{2}(4 \pi / 5+18 \theta) \cos ^{2}(\pi / 5+6 \theta) \cos ^{2}(\pi / 5+2 \theta),
\end{aligned}
$$

for $t=5$. Again, we have excellent agreements between the numerical data and our analytical 

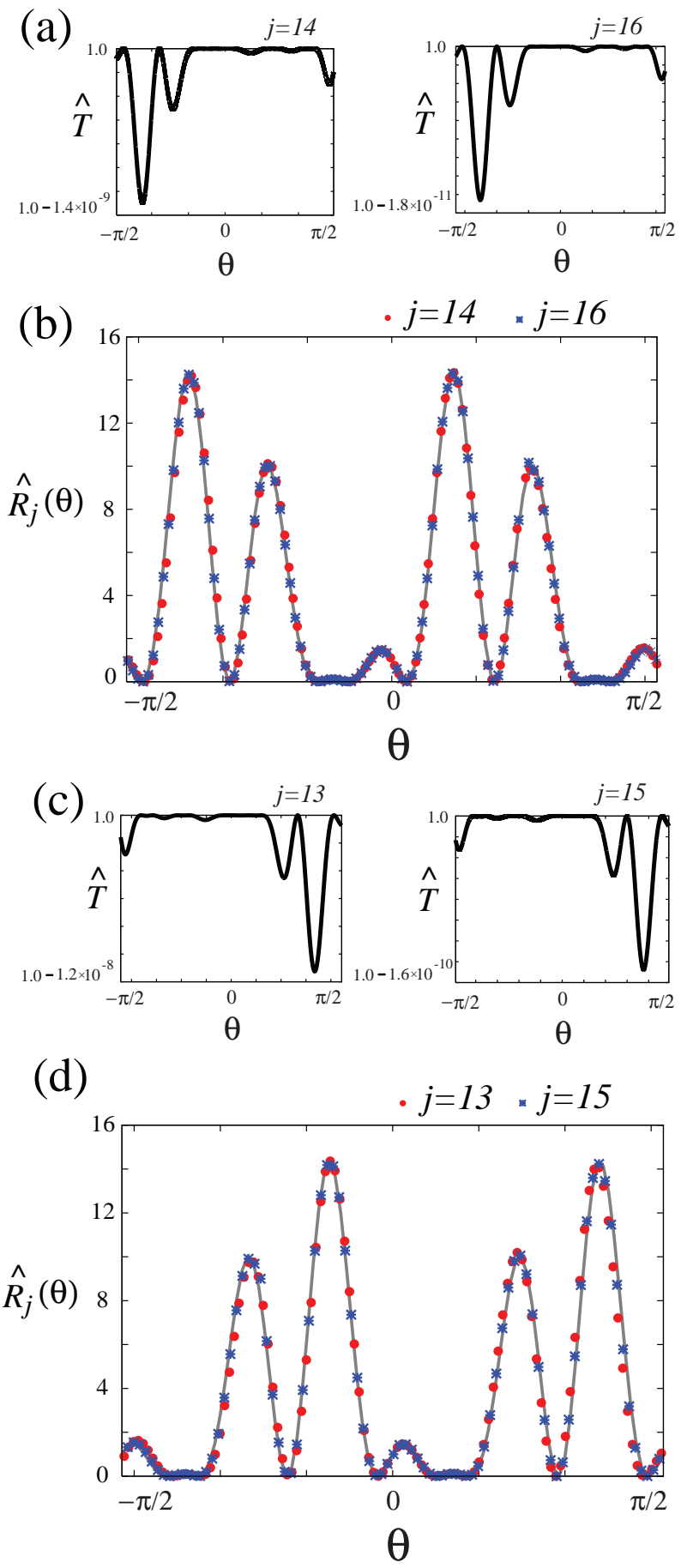

FIG. 15: (Color online) (a) Transmission coefficients $\hat{T}_{j}$ as functions of $\theta$ for $n_{\mathrm{A}}=3.0$ around $\kappa_{0}^{*}=$ $\pi / 3$. The generations are $j=14$ and $j=16$. (b) The ratio $\hat{R}_{j}(\theta)=\left(1-\hat{T}_{j}(\theta)\right) /\left(1-\hat{T}_{j-2}\left(\theta / 3^{2}\right)\right)$ for $n_{\mathrm{A}}=3.0$ around $\kappa_{0}^{*}=\pi / 3$. The generations are $j=14$ and $j=16$. The scaling function $f^{(1)}(\theta)$ is also plotted by the line. (c) $\hat{T}_{j}$ for the generations $j=13$ and $j=15$. (d) $\hat{R}_{j}$ for the generations $j=13$ and $j=15$. The scaling function is $f^{(2)}(\theta)$ in this case. 
(a)
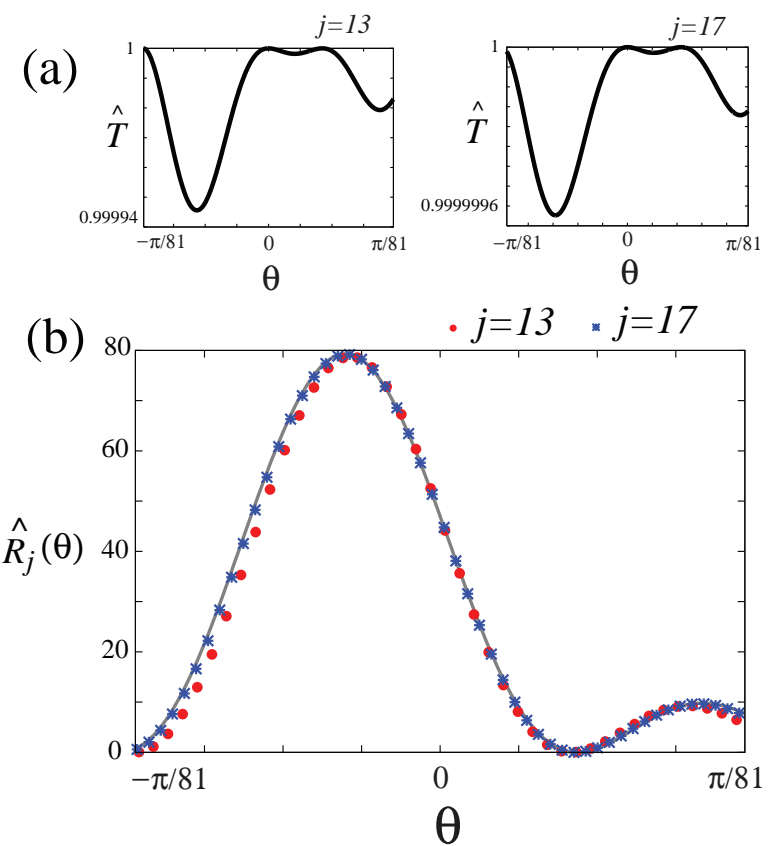

(c)
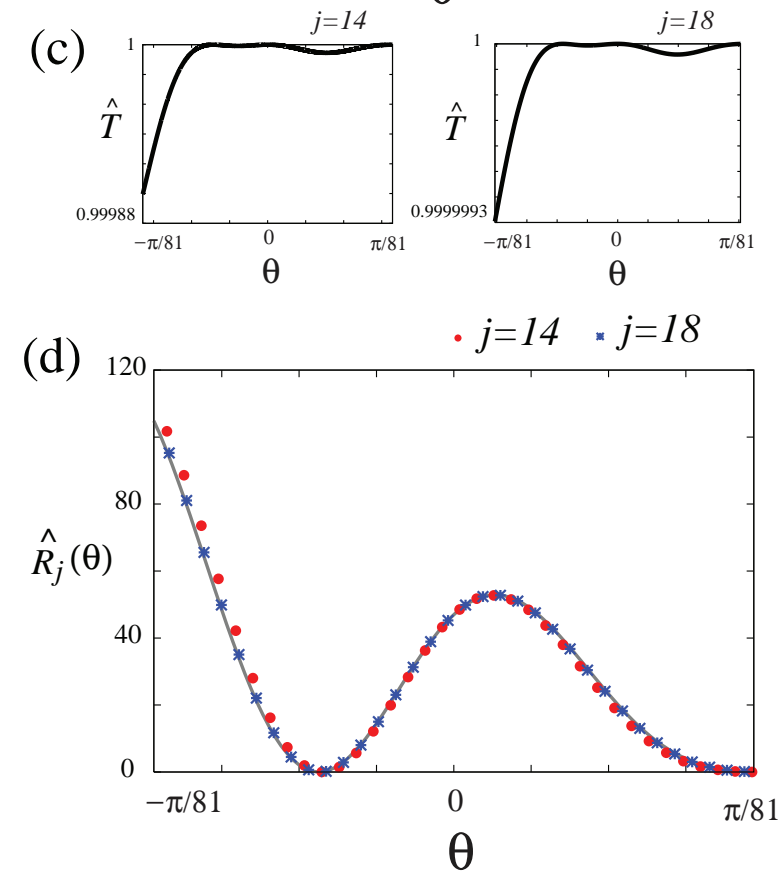

FIG. 16: (Color online) (a) Transmission coefficients $\hat{T}_{j}$ as functions of $\theta$ for $n_{\mathrm{A}}=5.0$ around $\kappa_{0}^{*}=$ $\pi / 5$. The generations are $j=13$ and $j=17$. (b) The ratio $\hat{R}_{j}(\theta)=\left(1-\hat{T}_{j}(\theta)\right) /\left(1-\hat{T}_{j-4}\left(\theta / 3^{4}\right)\right)$ for $n_{\mathrm{A}}=5.0$ around $\kappa_{0}^{*}=\pi / 5$. The generations are $j=13$ and $j=17$. The scaling function $f^{(2)}(\theta)$ is also plotted by the line. (c) $\hat{T}_{j}$ for the generations $j=14$ and $j=18$. (d) $\hat{R}_{j}$ for the generations $j=14$ and $j=18$. The scaling function is $f^{(3)}(\theta)$ in this case. 

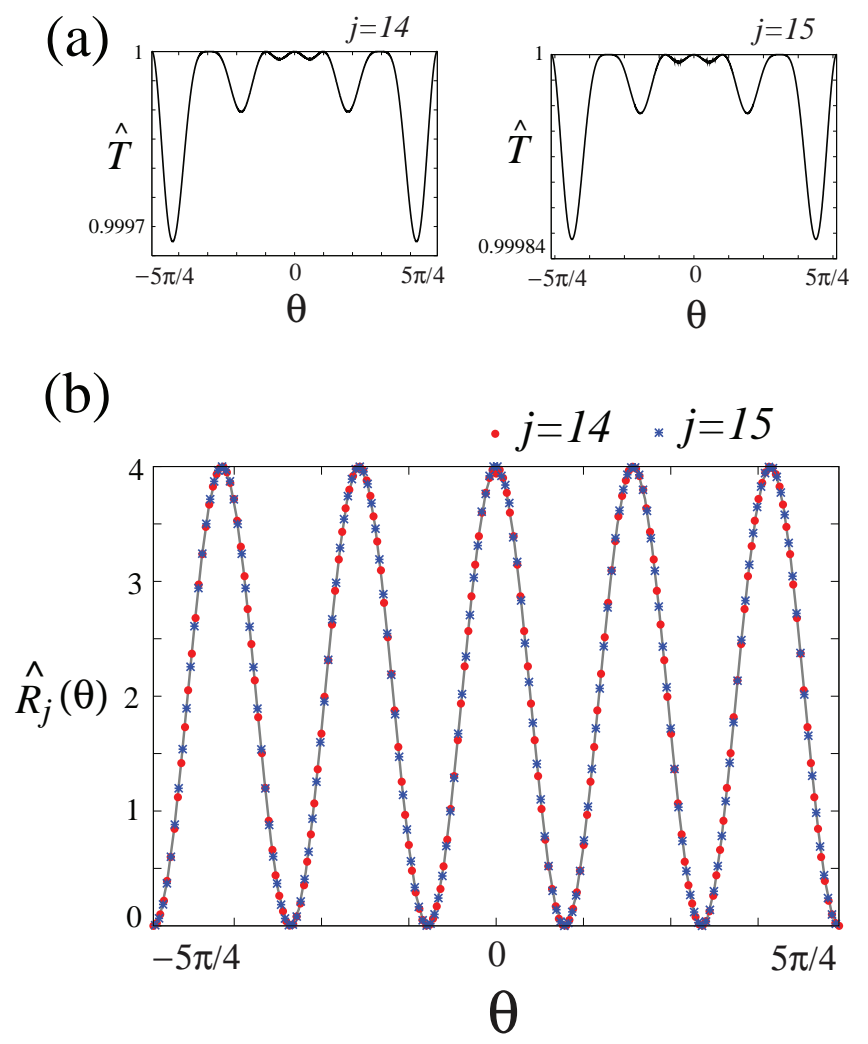

FIG. 17: (Color online) (a) Transmission coefficients $T_{j}$ as functions of $\theta$ for $n_{\mathrm{A}}=1 / 2$ around $\kappa_{1}^{*}=\pi$. The generations are $j=14$ and $j=15$. (b) The ratio $\hat{R}_{j}(\theta)=\left(1-\hat{T}_{j}(\theta)\right) /\left(1-\hat{T}_{j-1}(\theta / 3)\right)$ for $n_{\mathrm{A}}=1 / 2$ around $\kappa_{1}^{*}=\pi$. The generations are $j=14$ and $j=15$. The scaling function $f^{(1)}(\theta)$ is also plotted by the line.

results.

As an example with $l \neq 0$, we show a local scaling behavior for $n_{\mathrm{A}}=1 / 2$ and $\kappa_{1}^{*}=\pi$ in Fig.17. Here $p=1$ and the scaling function is given by

$$
f^{(1)}(\theta)=4 \cos ^{2}(2 \theta)
$$

It also reproduces the numerical data excellently.

We also confirm numerically that if complete transmission is achieved for a wave number with an irrational $\kappa^{*} / \pi$, no scaling behavior is obtained. The case of $n_{\mathrm{A}}=\sqrt{2}$ is shown in Fig 18. Although complete transmission is realized at $\kappa=\frac{\sqrt{2}}{2} \pi$, no scaling behavior of $T_{j}$ near the complete transmission is found in Fig, 18. 

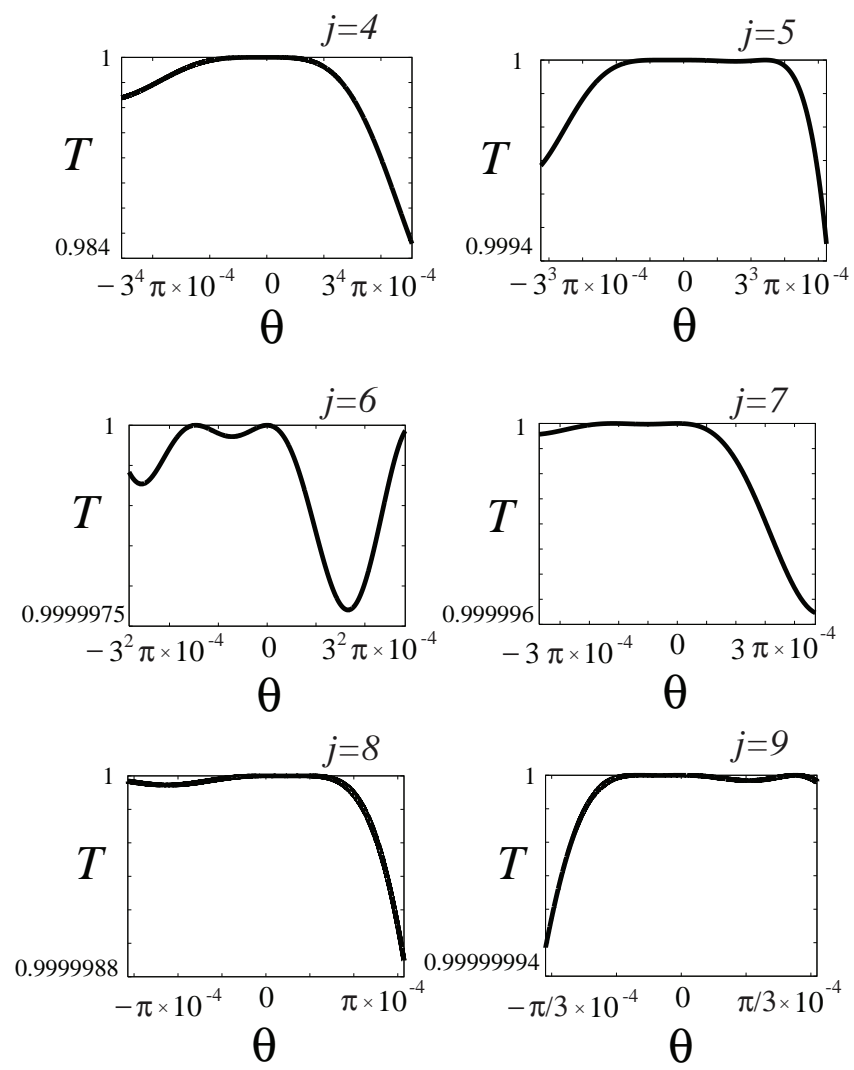

FIG. 18: Transmission coefficients $T_{j}$ as functions of $\theta$ for $n_{\mathrm{A}}=\sqrt{2}$ around $\kappa_{0}^{*}=\frac{\sqrt{2}}{2} \pi$. The generations are from $j=4$ to $j=9$. The horizontal axes are rescaled by a factor of three as a generation increases.

\section{SUMMARY AND DISCUSSIONS}

We investigate wave propagation through Cantor-set media on the basis of the renormalization-group equation. We analytically find that, for specific wave numbers, the transmission coefficients are governed by the logistic map. Especially, in the chaotic region, the transmission coefficients show sensitive dependence on small changes of parameters of the system such as the index of refraction. For wave numbers near the values giving the chaotic behavior, the transmission coefficients again show chaotic behaviors in the first few generations. For other values of wave numbers, our numerical results suggest that light transmits completely or reflects completely by the Cantor-set media. We also show that the transmission coefficients exhibit local scaling behaviors near complete transmission if the complete transmission is achieved at a wave number $\kappa^{*}$ with a rational $\kappa^{*} / \pi$. The analytic 
form of the scaling function is determined by $\kappa^{*}$ through the Euler's totient function.

Finally, we would like to mention that our approach developed here can be extended to the generalized Cantor sequences, where the length of each A becomes $1 / \beta$ as one increases the generation. For a positive integer $\beta$, in a similar manner as Sec $\amalg$ A , it is found that the initial wave numbers which give chaotic behaviors are

$$
\kappa=\frac{2 m}{(\beta-1)(\beta-2) \beta^{q}} \pi, \quad(m, q: \text { integers })
$$

In addition, for an odd positive integer $\beta$, chaotic behaviors are also found to appear for

$$
\kappa=\frac{2 m+1}{(\beta-1)(\beta-2) \beta^{q}} \pi, \quad(m, q: \text { integers }) .
$$

\section{APPENDIX A: PERIODICITY OF $\gamma_{i}^{(l)}\left(\kappa_{l}^{*}\right)$}

In this Appendix, we prove that $\gamma_{i}^{(l)}\left(\kappa_{l}^{*}\right)$ has the periodicity (158) if and only if $\kappa_{l}^{*} / \pi$ is a rational number. From the explicit forms of $\gamma_{i}^{(l)}$ given by (155), it is immediately found that if $\gamma_{i}^{(l)}\left(\kappa_{l}^{*}\right)$ has the periodicity (58), then $\kappa_{l}^{*} / \pi$ should be a rational number. Therefore, we will show in the following that if $\kappa_{l}^{*} / \pi$ is a rational number, then the periodicity (58) is obtained.

To prove this, we use the Euler's theorem:

$$
N^{\varphi(M)}=1, \quad(\bmod M)
$$

where $N$ and $M$ are mutually prime integers, and $\varphi(M)$ is the Euler's totient function which counts the number of positive integers not greater than and coprime to $M$ [25]. The Euler's totient function satisfies

$$
\varphi(n m)=\varphi(n) \varphi(m)
$$

for coprime positive integers $n$ and $m$.

Since $O\left(z_{l}\right)$ corrections in Eq. (55) disappear for $\kappa=\kappa_{l}^{*}, \gamma_{i}^{(l)}\left(\kappa_{l}^{*}\right)$ is given by

$$
\gamma_{i}^{(l)}\left(\kappa_{l}^{*}\right)=\left\{\begin{array}{ll}
\left(2 \cdot 3^{i}-2^{i-l+2} \cdot 3^{l-1}\right) \kappa_{l}^{*}, & \text { for } l \neq 0 \\
\left(2 \cdot 3^{i}-2^{i}\right) \kappa_{0}^{*}, & \text { for } l=0
\end{array}(\bmod \pi)\right.
$$


Thus for a rational $\kappa_{l}^{*} / \pi=s / t$ with coprime integers $s$ and $t$, we have

$$
\gamma_{i}^{(l)}\left(\kappa_{l}^{*}\right)=\left\{\begin{array}{ll}
\left(\frac{2 \cdot 3^{i}}{t}-\frac{2^{i-l+2} \cdot 3^{l-1}}{t}\right) s \pi, & \text { for } l \neq 0 \\
\left(\frac{2 \cdot 3^{i}}{t}-\frac{2^{i}}{t}\right) s \pi, & \text { for } l=0
\end{array} \quad(\bmod \pi) .\right.
$$

Now decompose $t$ into $t=2^{\eta} 3^{\xi} u$ where $\eta, \xi$ and $u$ are integers and $u$ is coprime to 2 and 3 . Then we obtain

$$
\gamma_{i}^{(l)}\left(\kappa_{l}^{*}\right)=\left\{\begin{array}{ll}
\left(\frac{3^{i-\xi}}{2^{\eta-1} u}-\frac{2^{i-l+2-\eta}}{3^{\xi-l+1} u}\right) s \pi, & \text { for } l \neq 0 \\
\left(\frac{3^{i-\xi}}{2^{\eta-1} u}-\frac{2^{i-\eta}}{3^{\xi} u}\right) s \pi, & \text { for } l=0
\end{array} \quad(\bmod \pi)\right.
$$

Since 3 and $2^{\eta} u$, and 2 and $3^{\xi} u$ are mutually prime integers, respectively, we have from the Euler's theorem

$$
\begin{aligned}
& 3^{\varphi\left(2^{\eta} u\right)}=1, \quad\left(\bmod 2^{\eta} u\right), \\
& 2^{\varphi\left(3^{\xi} u\right)}=1, \quad\left(\bmod 3^{\xi} u\right) .
\end{aligned}
$$

Moreover, using the relation (A2), we find

$$
\begin{aligned}
& 3^{\varphi\left(2^{\eta} 3^{\xi} u\right)}=\left(3^{\varphi\left(2^{\eta} u\right)}\right)^{\varphi\left(3^{\xi}\right)}=1, \quad\left(\bmod 2^{\eta} u\right), \\
& 2^{\varphi\left(2^{\eta} 3^{\xi} u\right)}=\left(2^{\varphi\left(3^{\xi} u\right)}\right)^{\varphi\left(2^{\eta}\right)}=1, \quad\left(\bmod 3^{\xi} u\right),
\end{aligned}
$$

namely

$$
3^{\varphi(t)}=1+2^{\eta} u M, \quad 2^{\varphi(t)}=1+3^{\xi} u N
$$

with integers $M$ and $N$. From (A8), we have

$$
\begin{aligned}
\gamma_{i+\varphi(t)}^{(l)}\left(\kappa_{l}^{*}\right) & =\left(\frac{3^{i+\varphi(t)-\xi}}{2^{\eta-1} u}-\frac{2^{i+\varphi(t)-l+2-\eta}}{3^{\xi-l+1} u}\right) s \pi \\
& =\left(\frac{3^{i-\xi}}{2^{\eta-1} u}-\frac{2^{i-l+2-\eta}}{3^{\xi-l+1} u}\right) s \pi+\left(2 \cdot 3^{i-\xi} M-2^{i-l+2-\eta} 3^{l-1} N\right) s \pi \\
& =\gamma_{i}^{(l)}\left(\kappa_{l}^{*}\right)+\left(2 \cdot 3^{i-\xi} M-2^{i-l+2-\eta} 3^{l-1} N\right) s \pi,
\end{aligned}
$$

for $l \neq 0$, and

$$
\begin{aligned}
\gamma_{i+\varphi(t)}^{(l)}\left(\kappa_{l}^{*}\right) & =\left(\frac{3^{i+\varphi(t)-\xi}}{2^{\eta-1} u}-\frac{2^{i+\varphi(t)-\eta}}{3^{\xi} u}\right) s \pi \\
& =\left(\frac{3^{i-\xi}}{2^{\eta-1} u}-\frac{2^{i-\eta}}{3^{\xi} u}\right) s \pi+\left(2 \cdot 3^{i-\xi} M-2^{i-\eta} N\right) s \pi \\
& =\gamma_{i}^{(l)}\left(\kappa_{l}^{*}\right)+\left(2 \cdot 3^{i-\xi} M-2^{i-\eta} N\right) s \pi
\end{aligned}
$$

for $l=0$. Therefore, we obtain Eq.(58) with $p=\varphi(t)$ for a sufficiently large $i$. Here note that $\varphi(t)$ is not the minimal period of $\gamma_{i}^{(l)}\left(\kappa_{l}^{*}\right)$ in general. Thus the minimal period $p$ is a divisor of $\varphi(t)$. 


\section{ACKNOWLEDGMENTS}

We are grateful to M. Yamanaka for useful discussions. This work was supported in part by Global COE Program "the Physical Sciences Frontier", MEXT, Japan for K.E.

[1] V. V. Konotop, O. I. Yordanov, and I. V. Yurkevich, Europhys. Lett. 12, 481(1990).

[2] X. Sun and D.L. Jaggard, J.Appl.Phys. 70, 2500(1991).

[3] M. Bertolotti, P. Masciulli, and C. Sibilia, Opt. Lett. 19, 777(1994)

[4] M. Bertolotti, P. Masciulli, C. Sibilia, F. Wijnands, and H. Hoekstra, J.Opt. Soc. Am.B 13, 628(1996).

[5] A.V. Lavrinenko, S.V.Zhukovsky, K.S. Sandomirski, and S.V. Gaponenko, Phys. Rev. E. 65, 036621(2002).

[6] F. Chiadini, V. Fiumara, I. M. Pinto, and A. Scaglione, Microw. Opt. Tech. Lett. 37, 339 (2003).

[7] M. Yamanaka and M. Kohmoto, cond-mat/0410239.

[8] N. Hatano, J. Phys. Soc. Jpn. 74, 3093(2005).

[9] U. Sangawa, IEICE Trans. Electron. E88-C, 1981 (2005).

[10] S. Sengupta, A. Chakrabarti, and S. Chattopadhyay, Physica B 344, 307 (2004)

[11] S. Sengupta, A. Chakrabarti, and S. Chattopadhyay Phys. Rev. B 71, 134204 (2005).

[12] M. Kohmoto, L.P. Kadanoff, and C. Tang, Phys. Rev. Lett. 50, 1870(1983).

[13] M. Kohmoto and Y. Oono, Phys. Lett. 102A, 145(1984).

[14] M. Kohmoto, B. Sutherland, and C. Tang, Phys. Rev. B. 35, 1020(1987).

[15] M. Kohmoto, B. Sutherland, and K. Iguchi, Phys. Rev. Lett. 58, 2436(1987).

[16] W. Gellermann, M. Kohmoto, B. Sutherland, and P.C. Taylor, Phys. Rev. Lett. 72, 633(1994).

[17] T. Hattori, N. Tsurumachi, S. Kawato, and H. Nakatsuka, Phys. Rev. B.50, 4220(1994).

[18] M.W. Takeda, S. Kirihara, Y. Miyamoto, K. Sakoda, and K. Honda, Phys. Rev. Lett. 92, 093902(2004).

[19] See, e. g., K. T. Alligood, T. D. Sauer, and J. A. Yorke, Chaos: An Introduction to Dynamical Systems (Springer, New York, 1996).

[20] T. Y. Li and J. A. Yorke, Am. Math. Monthly. 82, 985 (1975). 
[21] R. M. May, Nature. 261, 459 (1976).

[22] M. J. Feigenbaum, J. Stat. Phys. 19, 25 (1978).

[23] M. J. Feigenbaum, J. Stat. Phys. 21, 669 (1979).

[24] See, e.g., L. P. Kadanoff, Statistical Physics: Statics, Dynamics, and Renormalization (World Scientific, Singapore, 2000).

[25] M. Abramowitz and I. A. Stegun, Handbook of Mathematical Functions (Dover publications, New York, 1964), paragraph 24.3.2.

[26] Layers $\mathrm{B}$ in $\mathrm{C}_{j}$ have $j$ different lengths, $1 / 3^{j}, 1 / 3^{j-1}, \cdots 1 / 3$, thus in general they give $j$ different transfer matrices, $\mathcal{T}(\kappa), \mathcal{T}(3 \kappa), \cdots \mathcal{T}\left(3^{j-1} \kappa\right)$. But for $\kappa=\left(n / 3^{q}\right) \pi$ with $j \geq q, \mathcal{T}(\delta)$ 's in B's larger than $1 / 3^{j-q}$ are equal to $\mathcal{T}(n \pi)$. 